\title{
Multiplicative Chow-Künneth decompositions and varieties of cohomological K3 type
}

\author{
Lie $\mathrm{Fu}^{1,2} \cdot$ Robert Laterveer $^{3}$ (I) $\cdot$ Charles Vial $^{4}$
}

Received: 24 February 2020 / Accepted: 4 November 2020 / Published online: 1 June 2021

(C) The Author(s) 2021

\begin{abstract}
Given a smooth projective variety, a Chow-Künneth decomposition is called multiplicative if it is compatible with the intersection product. Following works of Beauville and Voisin, Shen and Vial conjectured that hyper-Kähler varieties admit a multiplicative Chow-Künneth decomposition. In this paper, based on the mysterious link between Fano varieties with cohomology of K3 type and hyper-Kähler varieties, we ask whether Fano varieties with cohomology of K3 type also admit a multiplicative Chow-Künneth decomposition, and provide evidence by establishing their existence for cubic fourfolds and Küchle fourfolds of type $c 7$. The main input in the cubic hypersurface case is the Franchetta property for the square of the Fano variety of lines; this was established in our earlier work in the fourfold case and is generalized here to arbitrary dimension. On the other end of the spectrum, we also give evidence that varieties with ample canonical class and with cohomology of K3 type might admit a multiplicative Chow-Künneth decomposition, by establishing this for two families of Todorov surfaces.
\end{abstract}

Keywords Algebraic cycles · Chow groups - Motives · K3 surfaces · Cubic hypersurfaces · Fano varieties of lines · Franchetta conjecture $\cdot$ Hyper-Kähler varieties $\cdot$ Beauville "splitting property" conjecture $\cdot$ Multiplicative Chow-Künneth decomposition

Mathematics Subject Classification $14 \mathrm{C} 15 \cdot 14 \mathrm{C} 25 \cdot 14 \mathrm{C} 30 \cdot 14 \mathrm{~J} 45 \cdot 14 \mathrm{~J} 42$

Lie Fu

fu@math.univ-lyon1.fr

Robert Laterveer

laterv@math.unistra.fr

Charles Vial

vial@math.uni-bielefeld.de

1 Institut Camille Jordan, Université Claude Bernard Lyon 1, Lyon, France

2 IMAPP, Radboud University, Nijmegen, The Netherlands

3 CNRS-IRMA, Université de Strasbourg, Strasbourg, France

4 Fakultät für Mathematik, Universität Bielefeld, Bielefeld, Germany 


\section{Introduction}

\subsection{Multiplicative Chow-Künneth decompositions}

Let $X$ be a smooth projective variety over a field $k$. A multiplicative Chow-Künneth decomposition-abbreviated MCK decomposition-for $X$ is a decomposition of the Chow motive of $X$, considered as an algebra object, that lifts the Künneth decomposition of its homological motive (when it exists). This notion was first introduced in [61] as a way to make explicitly verifiable the splitting principle for hyper-Kähler varieties due to Beauville [3]. Having a multiplicative Chow-Künneth decomposition is a restrictive condition on the variety $X$ and determining the class of varieties that could admit an MCK decomposition is still elusive. A precise definition of MCK decomposition is given in Sect. 2 and examples of varieties admitting or not admitting MCK decompositions are reviewed. Nonetheless, Beauville's splitting principle suggests that the situation for hyper-Kähler varieties is special.

Conjecture 1.1 [61] Any hyper-Kähler variety admits a multiplicative Chow-Künneth decomposition.

In fact, it could be moreover expected that, if it exists, such an MCK decomposition is unique for hyper-Kähler varieties. So far, Conjecture 1.1 has been established in the hyper-Kähler world for K3 surfaces [5] (reinterpreted by [61, Prop. 8.14]), Hilbert schemes of points on K3 surfaces [70] (see also [49]), generalized Kummer varieties [20] and all hyper-Kähler varieties birationally equivalent to these examples by [56].

\subsection{Fano varieties of cohomological K3 type}

A smooth projective complex variety is said to be of cohomological $K 3$ type, or more succinctly of $K 3$ type, if it is even-dimensional, say $\operatorname{dim} X=2 \mathrm{~m}$, and the Hodge numbers $h^{p, q}(X)$ are 0 for all $p \neq q$ except for $h^{m-1, m+1}(X)=h^{m+1, m-1}(X)=1$. Since the foundational work of Beauville-Donagi [2], it has become clear that hyper-Kähler varieties are intimately related to Fano varieties of cohomological K3 type. The folklore expectation seems to be that to any Fano variety of K3 type can be associated geometrically (via a moduli construction) a hyper-Kähler variety, and that the transcendental part of the middle cohomology of the Fano variety corresponds to the transcendental part of the second cohomology of the hyper-Kähler variety (via an Abel-Jacobi isomorphism). Apart from the example of Beauville-Donagi [2], this "folklore expectation" is based on the examples $[11,14,24-26,43,44,50]$. It is further motivated by the construction of a closed holomorphic 2-form on the non-singular locus of moduli spaces associated with any Fano variety of cohomological K3 type [34].

In this paper, we give further evidence that the conjectural existence of an MCK decomposition for hyper-Kähler varieties could transfer to Fano varieties of K3 type, and therefore strengthen the apparent connection between those two types of varieties. This question was already tackled for certain Fano varieties of K3 type in [38-41], where the so-called Franchetta property (cf. Definition 5.1) plays a crucial role. In our previous work [16], we established the Franchetta property for the Fano variety of lines on a cubic fourfold and its square. Here, we provide a generalization to smooth cubic hypersurfaces of any dimension: 
Theorem 1.2 (See Theorem 5.2) Let $B$ be the open subset of $\mathbb{P H}^{0}\left(\mathbb{P}^{n+1}, \mathcal{O}(3)\right)$ parameterizing smooth cubic hypersurfaces, and let $\mathcal{F} \rightarrow B$ be the universal family of their Fano varieties of lines. Then the families $\mathcal{F} \rightarrow B$ and $\mathcal{F} \times{ }_{B} \mathcal{F} \rightarrow B$ have the Franchetta property: For all fibers $F$ of $\mathcal{F} \rightarrow B$, the images of the restriction maps

$$
\mathrm{CH}^{*}(\mathcal{F}) \rightarrow \mathrm{CH}^{*}(F) \text { and } \mathrm{CH}^{*}\left(\mathcal{F} \times{ }_{B} \mathcal{F}\right) \rightarrow \mathrm{CH}^{*}(F \times F)
$$

inject, via the cycle class map, in the cohomology rings $\mathrm{H}^{*}(F, \mathbb{Q})$ and $\mathrm{H}^{*}(F \times F, \mathbb{Q})$, respectively.

As a consequence, we deduce the following, which can also be obtained from previous results of Diaz [13]:

Corollary 1.3 [13] All smooth cubic hypersurfaces have a multiplicative Chow-Künneth decomposition.

In particular, this applies to smooth cubic fourfolds, which are the Fano fourfolds that occur in the foundational work of Beauville-Donagi [2]. Corollary 1.3 is stated in a more precise form in Theorem 5.4 and is proved in Sect. 5.6, where we also explain the connection with the work of Diaz.

Finally, we obtain in Theorem 6.2 yet another positive answer for another type of Fano varieties of K3 type, namely Küchle fourfolds of type $c 7$. The method of proof also involves the Franchetta property.

\subsection{Varieties with ample canonical class and of cohomological K3 type}

On the other end of the spectrum, we also provide examples of varieties of K3 type with ample canonical class that admit an MCK decomposition:

Theorem 1.4 Let $S$ be a smooth Todorov surface with fundamental invariants $(0,9)$ or $(1$, 10). Then $S$ has a multiplicative Chow-Künneth decomposition.

The proof again involves, among other things, establishing the Franchetta property for some families. Theorem 1.4 gives the first example of a regular surface of general type with $p_{g} \neq 0$ that admits an MCK decomposition. For details on Todorov surfaces, we refer to Sect. 7.2 and to the references therein.

We are led to ask:

Question 1.5 Let $X$ be a smooth projective variety whose canonical divisor is ample or anti-ample. Assume that $X$ is of cohomological K3 type. Does $X$ have a multiplicative Chow-Künneth decomposition? If it exists, is it unique?

We note that without the assumption that $X$ be of cohomological K3 type, the question has a negative answer. In the case where the canonical divisor is ample, a very general curve of genus larger than 2 already provides an example that does not admit an MCK decomposition; see Example 3.3. Other examples are provided by very general surfaces in $\mathbb{P}^{3}$ of degree $\geq 7$; see Proposition 4.4. In the case where the canonical divisor is anti-ample, 
examples are provided by Beauville's examples of Fano threefolds that do not satisfy the so-called weak splitting property; see Example 2.11.

\subsection{Organization of the paper}

We start in Sect. 2 by reviewing the notion of MCK decomposition and examples of varieties for which such an MCK decomposition exists. The case of curves and regular surfaces is then extensively reviewed in Sects. 3 and 4, respectively. The results of Sects. 2, 3 and 4 are mostly expository and serve as motivation for the special role that hyper-Kähler varieties and varieties of $\mathrm{K} 3$ type with ample or anti-ample canonical class play with respect to MCK decompositions. Our new results are contained in the subsequent Sects. 5, 6 and 7 where we establish the existence of an MCK decomposition for smooth cubic hypersurfaces, Küchle fourfolds of type $c 7$ and certain Todorov surfaces, respectively.

\subsection{Future work}

In concomitant work, we use Corollary 1.3 on the existence of an MCK decomposition for smooth cubic fourfolds to establish in [17] the generalized Franchetta conjecture for Lehn-Lehn-Sorger-van Straten hyper-Kähler eightfolds and to study in [19] the Chow motives, as algebra objects, of smooth cubic fourfolds with Fourier-Mukai equivalent Kuznetsov categories. Furthermore, we will use Theorem 1.2 in [17] to compute the Chow motive of the Fano variety of lines on a smooth cubic hypersurface in terms of the Chow motive of the cubic hypersurface.

\subsection{Notation and conventions}

In this note, the word variety will refer to a reduced irreducible separated scheme of finite type over $\mathbb{C}$. We will write $\mathrm{H}^{j}(X)$ to indicate its rational singular cohomology group $\mathrm{H}^{j}(X(\mathbb{C}), \mathbb{Q})$. For a scheme of finite type over a field, $\mathrm{CH}^{i}(X)$ denotes the Chow group of codimension $i$ cycle classes on $X$ with rational coefficients. The category of rational Chow motives (pure motives with respect to rational equivalence as in [1]) is denoted by $\mathcal{M}_{\text {rat }}$, which is a pseudo-abelian rigid tensor category, whose tensor unit is denoted by $\mathbb{1}$. The contravariant functor from the category of smooth projective varieties to $\mathcal{M}_{\text {rat }}$ that sends a variety to its Chow motive is denoted by $\mathfrak{h}$.

\section{Generalities on multiplicative Chow-Künneth decompositions}

\subsection{Chow-Künneth decomposition}

Definition 2.1 (Chow-Künneth decomposition) Let $X$ be a smooth projective variety of dimension $d$. A Chow-Künneth decomposition for $X$ is a direct-sum decomposition

$$
\mathfrak{h}(X)=\mathfrak{h}^{0}(X) \oplus \cdots \oplus \mathfrak{h}^{2 d}(X)
$$

of its rational Chow motive in $\mathcal{M}_{\text {rat }}$, such that for any $0 \leq i \leq 2 d$, the Betti realization $\mathrm{H}^{*}\left(\mathfrak{h}^{i}(X)\right)=\mathrm{H}^{i}(X)$. 
In other words, a Chow-Künneth decomposition is a system of self-correspondences $\left\{\pi^{0}, \ldots, \pi^{2 d}\right\}$ in $\mathrm{CH}^{d}(X \times X)$ satisfying the following properties:

- (Projectors) $\pi^{i} \circ \pi^{i}=\pi^{i}$ for any $i$;

- (Orthogonality) $\pi^{i} \circ \pi^{j}=0$ for any $i \neq j$;

- (Completeness) $\pi^{0}+\cdots+\pi^{2 d}=\Delta_{X}$;

- (Künneth property) $\pi_{*}^{i} \mathrm{H}^{*}(X)=\mathrm{H}^{i}(X)$ for any $i$.

The existence of a Chow-Künneth decomposition for any smooth projective variety is part of Murre's conjectures [48].

Remark $2.2\left(\pi^{0}\right.$ and $\pi^{2 d}$ ) In a Chow-Künneth decomposition of a $d$-dimensional irreducible smooth projective variety $X$, the first and the last projectors are usually taken to be of the form $\pi^{0}=z \times 1_{X}$ and $\pi^{2 d}=1_{X} \times z^{\prime}$, respectively, where $z, z^{\prime}$ are 0 -cycles of degree 1 and $1_{X}$ is the fundamental class. We point out that if $X$ is Kimura finite-dimensional [29], we have $\mathfrak{h}^{0}(X) \simeq \mathbb{1}$ and $\mathfrak{h}^{2 d}(X) \simeq \mathbb{1}(-d)$; therefore, $\pi^{0}$ and $\pi^{2 d}$ must be of the above form.

Remark 2.3 (Duality) Thanks to the motivic Poincaré duality $\mathfrak{h}(X)=\mathfrak{h}(X)^{\vee}(-d)$, we see that a Chow-Künneth decomposition

$$
\mathfrak{h}(X)=\mathfrak{h}^{0}(X) \oplus \cdots \oplus \mathfrak{h}^{2 d}(X),
$$

naturally admits a dual decomposition:

$$
\mathfrak{h}(X)=\mathfrak{h}^{2 d}(X)^{\vee}(-d) \oplus \cdots \oplus \mathfrak{h}^{0}(X)^{\vee}(-d) .
$$

In terms of projectors, the dual of a system $\left\{\pi^{0}, \ldots, \pi^{2 d}\right\}$ is $\left\{{ }^{t} \pi^{2 d}, \ldots,{ }^{t} \pi^{0}\right\}$. A Chow-Künneth decomposition is called self-dual if for any $0 \leq i \leq 2 d$, we have $\mathfrak{h}^{i}(X)^{\vee}=\mathfrak{h}^{2 d-i}(X)(d)$, or equivalently, $\pi^{i}={ }^{t} \pi^{2 d-i}$.

\subsection{Murre's conjectures and the Bloch-Beilinson filtration}

Conjecture 2.4 [48] Let $X$ be a smooth projective variety of dimension $d$. Then

(A) there exists a Chow-Künneth decomposition $\left\{\pi^{0}, \ldots, \pi^{2 d}\right\}$. Any such decomposition induces a descending filtration

$$
F^{j} \mathrm{CH}^{i}(X):=\bigcap_{k>2 i-j} \operatorname{ker}\left(\pi_{*}^{k}: \mathrm{CH}^{i}(X) \rightarrow \mathrm{CH}^{i}(X)\right)=\sum_{k \leq 2 i-j} \operatorname{im}\left(\pi_{*}^{k}: \mathrm{CH}^{i}(X) \rightarrow \mathrm{CH}^{i}(X)\right)
$$

with the following properties:

(B) $F^{0} \mathrm{CH}^{i}(X)=\mathrm{CH}^{i}(X)$ and (B') $F^{i+1} \mathrm{CH}^{i}(X)=0$.

(C) The filtration $F^{\circ}$ on $\mathrm{CH}^{i}(X)$ is independent of the choice of the Chow-Künneth decomposition.

(D) $F^{1} \mathrm{CH}^{i}(X)=\mathrm{CH}^{i}(X)_{\mathrm{hom}}$.

We note that Murre's conjecture for all smooth projective varieties is equivalent to the existence of the Bloch-Beilinson filtration; see [28] for a precise statement. In 
particular, any Chow-Künneth decomposition induces a splitting of the conjectural Bloch-Beilinson filtration. As will be explained in Remark 2.8, the following notion of multiplicative Chow-Künneth decomposition gives a sufficient condition for the above splitting to be compatible with intersection product.

\subsection{Multiplicative Chow-Künneth decomposition}

Recall that if $X$ is a $d$-dimensional irreducible smooth scheme of finite type over a field, intersection product defines a (graded) ring structure on $\mathrm{CH}^{*}(X)=\bigoplus_{i} \mathrm{CH}^{i}(X)$; moreover, if $X$ is in addition proper, the intersection product is controlled by the class of the small diagonal $\delta_{X}:=\left\{(x, x, x) \in X^{3}\right\}$ in $\mathrm{CH}^{2 d}(X \times X \times X)$ in the sense that $\left(\delta_{X}\right)_{*}(\alpha \times \beta)=\alpha \cdot \beta$ for all $\alpha$ and $\beta \in \mathrm{CH}^{*}(X)$, where $\delta_{X}$ is viewed as a correspondence from $X \times X$ to $X$, or equivalently as a morphism $\mathfrak{h}(X \times X) \rightarrow \mathfrak{h}(X)$. Together with the canonical isomorphism $\mathfrak{h}(X \times X) \simeq \mathfrak{h}(X) \otimes \mathfrak{h}(X)$, the small diagonal $\delta_{X}$ endows the Chow motive $\mathfrak{h}(X)$ with the structure of a unital commutative algebra object. (The unit is the fundamental class of $X$, seen as a morphism $\mathbb{1} \rightarrow \mathfrak{h}(X)$.) We write

$$
\mu: \mathfrak{h}(X) \otimes \mathfrak{h}(X) \rightarrow \mathfrak{h}(X)
$$

for the multiplication thus defined.

Definition 2.5 (Multiplicative Chow-Künneth (MCK) decomposition, Shen-Vial [61]) Let $X$ be a smooth projective variety of dimension $d$. A Chow-Künneth decomposition

$$
\mathfrak{h}(X)=\mathfrak{h}^{0}(X) \oplus \cdots \oplus \mathfrak{h}^{2 d}(X)
$$

is called multiplicative, if for any $0 \leq i, j \leq 2 d$, the restriction of the multiplication $\mu: \mathfrak{h}(X) \otimes \mathfrak{h}(X) \rightarrow \mathfrak{h}(X)$ to the direct summand $\mathfrak{h}^{i}(X) \otimes \mathfrak{h}^{j}(X)$ factors through the direct summand $\mathfrak{h}^{i+j}(X)$.

Note that a Chow-Künneth decomposition is always multiplicative modulo homological equivalence; the key point is to require this property modulo rational equivalence. In practice, it is useful to express the above notion in terms of projectors and correspondences.

Lemma 2.6 Let $X$ be a smooth projective variety of dimension $d$. Let $\left\{\pi^{0}, \ldots, \pi^{2 d}\right\}$ be the system of projectors corresponding to a Chow-Künneth decomposition of X (see Definition 2.1). Then the following conditions are equivalent:

(i) The Chow-Künneth decomposition is multiplicative.

(ii) For any $i, j, k$ such that $i+j \neq k$, we have $\pi^{k} \circ \delta_{X} \circ\left(\pi^{i} \otimes \pi^{j}\right)=0$.

(iii) For any $i, j$, we have $\pi^{i+j} \circ \delta_{X} \circ\left(\pi^{i} \otimes \pi^{j}\right)=\delta_{X} \circ\left(\pi^{i} \otimes \pi^{j}\right)$.

(iv) $\delta_{X}=\sum_{i, j} \pi^{i+j} \circ \delta_{X} \circ\left(\pi^{i} \otimes \pi^{j}\right)$.

Here, $\delta_{X}$ denotes the small diagonal of $X^{3}$, viewed as a correspondence from $X \times X$ to $X$. Proof Noting that $\mu$ is induced by $\delta_{X}$ by definition, the equivalence between $(i)$ and (ii) becomes tautological.

$(i i) \Longrightarrow(i v)$ : by the completeness of the system $\sum_{i} \pi^{i}=\Delta_{X}$, we see that 


$$
\begin{aligned}
\delta_{X} & =\sum_{i, j, k} \pi^{k} \circ \delta_{X} \circ\left(\pi^{i} \otimes \pi^{j}\right) \\
& =\sum_{k=i+j} \pi^{k} \circ \delta_{X} \circ\left(\pi^{i} \otimes \pi^{j}\right)+\sum_{k \neq i+j} \pi^{k} \circ \delta_{X} \circ\left(\pi^{i} \otimes \pi^{j}\right) \\
& =\sum_{i, j} \pi^{i+j} \circ \delta_{X} \circ\left(\pi^{i} \otimes \pi^{j}\right) .
\end{aligned}
$$

(iv) $\Longrightarrow\left(\right.$ iii): it is enough to post-compose both sides of (iv) with $\pi^{i} \otimes \pi^{j}$ and use the orthogonality between the projectors.

(iii) $\Longrightarrow$ (ii): it suffices to pre-compose both sides of (iii) with $\pi^{k}$ and use the orthogonality between the projectors.

It turns out that an MCK decomposition is automatically self-dual (Remark 2.3):

Proposition 2.7 (Multiplicativity implies self-duality [18, §6 Footnote 24]). Let $\left\{\pi^{0}, \ldots, \pi^{2 d}\right\}$ be a multiplicative Chow-Künneth decomposition for a smooth projective variety $X$ of dimension $d$. Then it is self-dual, that is, $\pi^{i}={ }^{t} \pi^{2 d-i}$ for all $i$.

Proof Projecting both sides of (iv) in Lemma 2.6 to the first two factors (or equivalently, pre-composing with the canonical morphism $\epsilon: \mathfrak{h}(X) \rightarrow \mathbb{1}(-d)$ given by the fundamental class), one finds

$$
\epsilon \circ \delta_{X}=\sum_{i, j} \epsilon \circ \pi^{i+j} \circ \delta_{X} \circ\left(\pi^{i} \otimes \pi^{j}\right)
$$

As $\epsilon \circ \pi^{k}: \mathfrak{h}(X) \rightarrow \mathbb{1}(-d)$ is a rational multiple of the fundamental class for all $k$, we get that for any $i+j \neq 2 d, \epsilon \circ \pi^{i+j}=0$ and $\epsilon \circ \pi^{2 d}=\epsilon$. Therefore, the equality simplifies to

$$
\epsilon \circ \delta_{X}=\sum_{i} \epsilon \circ \delta_{X} \circ\left(\pi^{2 d-i} \otimes \pi^{i}\right)
$$

Now noting that $\epsilon \circ \delta_{X}$ is the diagonal class $\Delta_{X} \in \mathrm{CH}^{d}(X \times X)$, we obtain

$$
\Delta_{X}=\sum_{i}\left({ }^{t} \pi^{2 d-i} \otimes \pi^{i}\right)_{*}\left(\Delta_{X}\right)
$$

In other words, id $=\sum_{i}{ }^{t} \pi^{2 d-i} \circ \pi^{i}$. This allows us to conclude by composing with $\pi^{i}$ and ${ }^{t} \pi^{2 d-i}$ :

$$
\pi^{i}=\left(\sum_{j}{ }^{t} \pi^{2 d-j} \circ \pi^{j}\right) \circ \pi^{i}={ }^{t} \pi^{2 d-i} \circ \pi^{i}={ }^{t} \pi^{2 d-i} \circ\left(\sum_{j}{ }^{t} \pi^{2 d-j} \circ \pi^{j}\right)={ }^{t} \pi^{2 d-i} .
$$

Remark 2.8 (Multiplicative bigrading) A multiplicative Chow-Künneth decomposition $\mathfrak{h}(X)=\mathfrak{h}^{0}(X) \oplus \cdots \oplus \mathfrak{h}^{2 d}(X)$ naturally gives rise to a multiplicative bigrading on the Chow ring $\mathrm{CH}^{*}(X)=\bigoplus_{i, s} \mathrm{CH}^{i}(X)_{(s)}$ with

$$
\mathrm{CH}^{i}(X)_{(s)}:=\mathrm{CH}^{i}\left(\mathfrak{h}^{2 i-s}(X)\right):=\operatorname{Hom}\left(\mathbb{1}(-i), \mathfrak{h}^{2 i-s}(X)\right) .
$$

Here, the multiplicativity means that 


$$
\mathrm{CH}^{i}(X)_{(s)} \cdot \mathrm{CH}^{i^{\prime}}(X)_{\left(s^{\prime}\right)} \subseteq \mathrm{CH}^{i+i^{\prime}}(X)_{\left(s+s^{\prime}\right)},
$$

which clearly follows from the multiplicativity of the Chow-Künneth decomposition. However, we note that actually any Chow-Künneth decomposition induces a splitting of the Chow groups of $X$ and the property that it is a ring grading, i.e., that $\mathrm{CH}^{i}(X)_{(s)} \cdot \mathrm{CH}^{i^{\prime}}(X)_{\left(s^{\prime}\right)} \subseteq \mathrm{CH}^{i+i^{\prime}}(X)_{\left(s+s^{\prime}\right)}$ is strictly weaker than having a MCK decomposition; a very general curve of genus $\geq 2$ does not admit an MCK decomposition (Example 3.3), but any splitting of its Chow groups induced by a Chow-Künneth decomposition is compatible with the intersection product. The new grading is chosen so that, via Murre's conjecture $2.4(\mathrm{C})$,

$$
\mathrm{CH}^{i}(X)_{(s)}=\mathrm{Gr}_{F}^{s} \mathrm{CH}^{i}(X) .
$$

In other words, a multiplicative Chow-Künneth decomposition should induce a multiplicative splitting of the (conjectural) Bloch-Beilinson filtration on Chow groups.

We call the indexation by $s$ the grade of a cycle. For example, by Murre's conjecture 2.4 (B) and (D), all cycles of negative grade are expected to be zero and the subspace consisting of cycles of grade zero, $\mathrm{CH}^{*}(X)_{(0)}$, is expected to inject into the cohomology of $X$ via the cycle class map. In particular, the subalgebra of $\mathrm{CH}^{*}(X)$ generated by $\mathrm{CH}^{1}(X)_{(0)}$ is expected to inject into cohomology; this is Beauville's so-called weak splitting property [3].

\subsection{Which varieties admit a multiplicative Chow-Künneth decomposition?}

Although a Chow-Künneth decomposition is conjectured to exist for all smooth projective varieties, there exist examples of varieties (in fact, examples of curves; see, e.g., Example 3.3) that do not admit any MCK decomposition. Nonetheless, as shown by Shen-Vial [60], the notion of multiplicative Chow-Künneth decomposition is robust enough to allow many standard procedures to produce new examples out of old ones.

Proposition 2.9 [60] Let $X$ and $Y$ be smooth projective varieties admitting MCK decompositions $\left\{\pi_{X}^{i}\right\}$ and $\left\{\pi_{Y}^{i}\right\}$, respectively.

- (Product) The product $X \times Y$ has a naturally induced MCK decomposition: for all $k$, $\pi_{X \times Y}^{k}:=\sum_{i=0}^{k} \pi_{X}^{i} \otimes \pi_{Y}^{k-i}$.

- (Projective bundle) If $E$ is a vector bundle on $X$ whose Chern classes are all of grade 0. then $\mathbb{P}(E)$ has a natural MCK decomposition.

- (Blow-up) Suppose that $Y$ is a subvariety of $X$ and that, as an element in $\mathrm{CH}(X \times Y)$, the graph of the embedding is of grade 0 for the natural product MCK decomposition. Assume further that the Chern classes of the normal bundle are of grade 0 and the Chern classes of the tangent bundle of $X$ are of grade 0 . Then the blow-up of $X$ along $Y$ admits a natural MCK decomposition.

- (Quotient) If a finite group $G$ acts on $X$ such that the graphs of the automorphisms (as elements in $\mathrm{CH}(X \times X))$ are of grade 0 , then the quotient $X / G$ admits a natural MCK decomposition. 
- (Hilbert scheme) Assume that the Chern classes of X are of grade 0. Then the Hilbert schemes of length-2 and length-3 subschemes of X admit an MCK.

Consequently, we may ask the following general, but vague, question:

Question 2.10 Which smooth projective varieties constitute the "building blocks" of varieties admitting an MCK decomposition ? For which of those can we expect an MCK decomposition to be unique?

Note that, since homological equivalence and rational equivalence agree on powers of a variety whose motive is of Lefschetz type (for example, toric varieties, homogeneous varieties), such a variety admits a unique Chow-Künneth decomposition and this decomposition is multiplicative.

The original and main motivation for studying MCK decompositions comes from the study of Chow rings of varieties with trivial canonical bundle. A canonical multiplicative Chow-Künneth decomposition exists in the following cases: abelian varieties [4, 12, 31], K3 surfaces [5] (interpreted by [61, Proposition 8.4]) and some (conjecturally, all) hyper-Kähler varieties [16, 20, 61, 70]. The case of Calabi-Yau varieties is not so clear: there are examples of Calabi-Yau varieties due to Beauville [3, Example 2.1.5(b)] that do not admit a Chow-Künneth decomposition inducing a grading satisfying (1), while examples of Calabi-Yau varieties with a MCK decomposition exist in all dimensions [42]. Concerning the uniqueness of an MCK decomposition, we will show that this is the case for curves and for regular surfaces with finite-dimensional motive in the sense of Kimura. It is expected [18, 61] that for hyper-Kähler varieties, if an MCK decomposition exists, then it is unique. Note, however, that an MCK decomposition is not unique for abelian varieties; translating the canonical Chow-Künneth decomposition (which is multiplicative) of an abelian variety along a point that is not rationally equivalent to the origin provides a new MCK decomposition.

Concerning the existence of an MCK decomposition in general, the answer is for the time being not clear in general. The main purpose of the paper is, beyond reviewing known examples of varieties admitting or not admitting an MCK decomposition, to explore whether varieties with ample or anti-ample canonical class can be added to the list of varieties admitting such a decomposition.

In the case of Fano varieties, there are examples of varieties (with motive not of Lefschetz type) that admit an MCK decomposition. By [18, Proposition 5.7], all Fermat cubic hypersurfaces admit an MCK decomposition. It is in fact conjectured in [18, Conjecture 5.8] that all Fano or Calabi-Yau Fermat hypersurfaces admit MCK decompositions. Other examples have been exhibited in [38-41], and Corollary 1.3 shows that cubic fourfolds can be added to the list. On the other hand, there are also examples of Fano varieties that do not admit an MCK decomposition:

Example 2.11 (Fano varieties may fail to have an MCK decomposition) In [3, Example 2.1.5(a)], Beauville constructed, by blowing up $\mathbb{P}^{3}$ along certain smooth curves, examples of Fano threefolds such that the subalgebra generated by divisors does not inject in cohomology. Such Fano threefolds cannot have an MCK decomposition. Indeed, by [68, $\S 4.2 .2]$, any Chow-Künneth decomposition of the blow-up $X$ of a curve inside $\mathbb{P}^{3}$ will be such that $\mathrm{CH}^{1}(X)=\mathrm{CH}^{1}(X)_{(0)}$ and such that $\mathrm{CH}^{*}(X)_{(0)} \hookrightarrow \mathrm{H}^{*}(X)$ is injective. In particular, for any choice of Chow-Künneth decomposition, $X$ fails to satisfy Beauville's weak 
splitting property, and hence, by Remark 2.8 any choice of Chow-Künneth decomposition for $X$ fails to be multiplicative.

In the case of varieties with ample canonical bundle, we will review below that a very general curve of genus $>2$ (Example 3.3) does not admit an MCK decomposition. Moreover, Proposition 4.4 suggests that a general surface in $\mathbb{P}^{3}$ of degree $\geq 7$ also does not admit an MCK decomposition. There are, however, examples of varieties with ample canonical bundle (and with motive not of Lefschetz type) that admit an MCK decomposition: This is the case, for example, for any product of hyperelliptic curves of genus $>1$ by combining Example 3.2 with Proposition 2.9. Our Theorem 1.4 provides further examples, namely Todorov surfaces of type $(0,9)$ or $(1,10)$, that are not (birational to) products of curves.

It turns out that the Todorov surfaces we study are intimately linked to K3 surfaces. Likewise the Fano examples of [38-41], as well as the cubic fourfolds of Corollary 1.3, have cohomology of K3 type: these are Fano fourfolds with Hodge numbers $h^{p, q}=0$ for all $p \neq q$ except for $h^{3,1}(X)=h^{1,3}(X)=1$. In the light of these examples, but also based (in the Fano case) on the folklore expectation that to any Fano variety of K3 type can be associated geometrically (via a moduli construction) a hyper-Kähler variety, we ask whether every smooth projective variety with ample or anti-ample canonical bundle, and with cohomology of K3 type, admits a (unique) multiplicative Chow-Künneth decomposition; cf. Question 1.5.

\section{Curves}

\subsection{Multiplicative Chow-Künneth decomposition for curves}

As mentioned before, the projective line (a special case of homogeneous varieties) and elliptic curves (special cases of abelian varieties) admit canonical multiplicative Chow-Künneth decompositions. Let $C$ be a smooth projective curve of genus $g \geq 2$. As it is Kimura finite-dimensional, by Remark 2.2 and Proposition 2.7, a multiplicative Chow-Künneth decomposition for $C$ must take the following form:

$$
\pi^{0}=z \times 1_{C}, \pi^{1}=\Delta_{C}-z \times 1_{C}-1_{C} \times z, \pi^{2}=1_{C} \times z,
$$

where $z$ is a 0 -cycle of degree 1 and $1_{C}$ is the fundamental class. Given such a 0 -cycle $z$, there is the natural embedding $\imath: C \rightarrow J(C)$, which sends a point $p \in C$ to $\mathcal{O}_{C}(p-z)$. Denote $[C]:=\iota_{*}\left(1_{C}\right) \in \mathrm{CH}_{1}(J(C))$.

Proposition 3.1 (MCK decomposition for curves) Notation is as above $(g \geq 2)$. Let $z$ be a 0 -cycle of degree 1 on $C$. Then the following conditions are equivalent:

(i) the Chow-Künneth decomposition (2) determined by $z$ is multiplicative;

(ii) the modified small diagonal $\Gamma_{3}(C, z)=0$ in $\mathrm{CH}^{2}\left(C^{3}\right)$, where

$$
\begin{aligned}
\Gamma_{3}(C, z):= & \delta_{C}-p_{12}^{*}\left(\Delta_{C}\right) p_{3}^{*}(z)-p_{23}^{*}\left(\Delta_{C}\right) p_{1}^{*}(z) \\
& -p_{13}^{*}\left(\Delta_{C}\right) p_{2}^{*}(z)+p_{1}^{*}(z) p_{2}^{*}(z)+p_{1}^{*}(z) p_{3}^{*}(z)+p_{2}^{*}(z) p_{3}^{*}(z) ;
\end{aligned}
$$

(iii) the class [C] belongs to $\mathrm{CH}_{1}(J(C))_{(0)}$. 
In particular, if it exists, an MCK decomposition for $C$ is unique, given by $z=\frac{1}{2 g-2} K_{C}$.

Proof The equivalence between ( $i$ ) and (ii) follows from a direct computation using Lemma 2.6 (see [61, Proposition 8.14]).

(ii) $\Longrightarrow$ (iii) is proved in [18, Proposition 7.1] using an idea from [5, Proposition 3.2]. Let $f: C^{3} \rightarrow J(C)$ be the composition of the embedding $\imath^{3}: C^{3} \rightarrow J(C)^{3}$ followed by the summation on $J(C)$. We have

$$
f_{*}\left(\Gamma_{3}(C, z)\right)=[3]_{*}[C]-3[2]_{*}[C]+3[C]=0 \quad \text { in } \mathrm{CH}_{1}(J(C)) .
$$

Using the Beauville decomposition [4] of $\mathrm{CH}_{1}(J(C))$, we see that [C] belongs to $\mathrm{CH}_{1}(J(C))_{(0)}$.

(iii) $\Longrightarrow($ i $)$ is implied by [18, Propositions 5.3 and 6.1]. Finally, for the uniqueness, letting (ii) act on $\Delta_{C}$, we get $c_{1}\left(T_{C}\right)=(2-2 g) z$. Hence, $z$ is determined by the curve.

The next two examples illustrate that Proposition 3.1 leads to both existence and nonexistence results on MCK decompositions for curves:

Example 3.2 (Curves with MCK decompositions [61, Example 8.16]) If $C$ is hyperelliptic, take $z$ to be a Weierstrass point, then Gross-Schoen [23] proved that the modified small diagonal $\Gamma_{3}(C, z)$ vanishes. Alternately, by [64, Proposition 2.1], the class [C] belongs to $\mathrm{CH}_{1}(J(C))_{(0)}$. By Proposition 3.1, this implies that the Chow-Künneth decomposition (2) is multiplicative.

Example 3.3 (Curves without MCK decompositions [18, §7]) As is pointed out before, any MCK decomposition for a curve $C$ is determined by a 0 -cycle of degree 1 . If $C$ admits an MCK decomposition, then Proposition 3.1 (iii) implies in particular that the Ceresa cycle $[C]-[-1]_{*}[C] \in \mathrm{CH}_{1}(J(C))$ vanishes.

Ceresa [10] proved that the Ceresa cycle of a very general complex curve of genus $>2$ is not algebraically trivial; as such, a very general complex curve of genus $>2$ does not admit an MCK decomposition.

As more explicit examples, Otsubo [53] proves that the Ceresa cycle of Fermat curves of degree $4 \leq d \leq 1000$ is not algebraically trivial. Therefore, those curves do not admit any MCK decomposition. A specialization argument (cf. [72, Lemma 3.2]) then establishes that a very general plane curve of degree $4 \leq d \leq 1000$ does not admit an MCK decomposition.

Remark 3.4 (MCK decomposition modulo algebraic equivalence) If instead of rational equivalence, we work with algebraic equivalence, the analogue of Proposition 3.1 still holds and the choice of the 0-cycle $z$ becomes irrelevant. More precisely, given a smooth projective curve $C$, the following conditions are equivalent:

(i) $C$ admits a multiplicative Chow-Künneth decomposition modulo algebraic equivalence (which, again, must be of the form (2)).

(ii) The modified small diagonal $\Gamma_{3}(C, \mathrm{pt})$ is algebraically trivial.

(iii) The class $[C]$ belongs to $\mathrm{CH}_{1}(J(C))_{(0)} / \mathrm{alg}$.

Of course, (iii) implies as before

(iv) the Ceresa cycle $[C]-[-1]_{*}[C]$ is algebraically trivial. 
Now the point of this remark is that (iv) is actually equivalent to (iii), hence also to $(i)$, or (ii). Indeed, in the Beauville decomposition

$$
\mathrm{CH}_{1}(J(C))=\bigoplus_{s=0}^{g-1} \mathrm{CH}_{1}(J(C))_{(s)},
$$

denote by $C_{(s)}$ the grade $s$ component of the class $[C]$. It is well-defined modulo algebraic equivalence. Then (iv) implies that $C_{(1)}=0$. By Marini's result [45, Corollary 26], we have $C_{(s)}$ is algebraically trivial for all $s>0$, that is, $[C] \in \mathrm{CH}_{1}(J(C))_{(0)} / \mathrm{alg}$.

In conclusion, the vanishing of the Ceresa cycle characterizes the multiplicativity of the Chow-Künneth decomposition modulo algebraic equivalence.

\subsection{On the tautological ring of powers of curves}

The following proposition, essentially due to Tavakol [64], relates for a given curve the existence of an MCK decomposition to the existence of enough relations in the tautological ring.

Proposition 3.5 Let $C$ be a smooth projective complex curve. Let $\mathrm{R}^{*}\left(C^{m}\right) \subseteq \mathrm{CH}^{*}\left(C^{m}\right)$ denote the subring generated by pullbacks of the canonical divisor and of the diagonal $\Delta_{C}$. Then $C$ admits an MCK decomposition if and only if the cycle class map induces injections

$$
\mathrm{R}^{i}\left(C^{m}\right) \hookrightarrow \mathrm{H}^{2 i}\left(C^{m}\right)
$$

for all positive integers $m$ and all $i$.

Proof Tavakol [64] shows that the cohomological relations among $p_{i}^{*} K_{C}$ and $p_{i j}^{*} \Delta_{C}$ are generated by three relations, namely the Faber-Pandharipande relation, the Gross-Schoen relation and a relation implied by the finite-dimensionality relation; see also [74, Remark 3.8(i)]. By Kimura [29], the finite-dimensionality relation holds modulo rational equivalence. The Gross-Schoen relation is nothing but the vanishing of the modified small diagonal. By Proposition 3.1, this relation is equivalent to the existence of an MCK decomposition. Finally, the Faber-Pandharipande relation, $p_{1}^{*} K_{C} \cdot p_{2}^{*} K_{C}=\operatorname{deg}\left(K_{C}\right) p_{1}^{*} K_{C} \cdot \Delta_{C}$, can be obtained by making both sides of the Gross-Schoen relation, viewed as correspondences from $C$ to $C \times C$, act on $K_{C}$.

\section{Regular surfaces}

\subsection{Multiplicative Chow-Künneth decomposition for regular surfaces}

Let $S$ be a regular surface, that is, a smooth projective complex surface with $\mathrm{H}^{1}\left(S, \mathcal{O}_{S}\right)=0$. Then for any 0-cycle of degree 1 on $S$, we have a self-dual Chow-Künneth decomposition

$$
\pi^{0}=z \times 1_{S}, \pi^{4}=1_{S} \times z, \pi^{2}=\Delta_{S}-\pi^{0}-\pi^{4}, \pi^{1}=\pi^{3}=0 .
$$


Assuming the Kimura finite-dimensionality conjecture [29], any self-dual Chow-Künneth decomposition should be of this form (Remark 2.2). Similarly to Proposition 3.1, we have the following result.

Proposition 4.1 (MCK decomposition for regular surfaces) Let $S$ be a regular smooth projective surface. Let $z$ be a 0 -cycle of degree 1 on $S$. Then the following conditions are equivalent:

(i) the Chow-Künneth decomposition (3) is multiplicative;

(ii) the modified small diagonal $\Gamma_{3}(S, z) \in \mathrm{CH}^{4}\left(S^{3}\right)$ vanishes, where

$$
\begin{aligned}
\Gamma_{3}(S, z):= & \delta_{S}-p_{12}^{*}\left(\Delta_{S}\right) p_{3}^{*}(z)-p_{23}^{*}\left(\Delta_{S}\right) p_{1}^{*}(z)-p_{13}^{*}\left(\Delta_{S}\right) p_{2}^{*}(z) \\
& +p_{1}^{*}(z) p_{2}^{*}(z)+p_{1}^{*}(z) p_{3}^{*}(z)+p_{2}^{*}(z) p_{3}^{*}(z) .
\end{aligned}
$$

Moreover, they imply the following two properties:

(iii) $\operatorname{Im}\left(\mathrm{CH}^{1}(S) \otimes \mathrm{CH}^{1}(S) \rightarrow \mathrm{CH}^{2}(S)\right)=\mathbb{Q} \cdot z$.

(iv) $c_{2}\left(T_{S}\right)=\chi_{\text {top }}(S) z$, where $\chi_{\text {top }}$ is the topological Euler characteristic.

In particular, if it exists, an MCK decomposition of the form (3) for $S$ is unique.

Proof The equivalence between ( $i$ ) and (ii) is a direct computation using Lemma 2.6, which was first observed in [61, Proposition 8.4]. The implication from them to (iii) and (iv) is proved as in [5]: Let both sides of (ii) act on the exterior product of two divisors to obtain (iii) and on $\Delta_{S}$ to get (iv).

Example 4.2 (Regular surfaces with an MCK decomposition) First we note that any Chow-Künneth decomposition of a surface whose Chow motive is of Lefschetz type is multiplicative. Hence, any complex surface with trivial Chow group of 0 -cycles admits an MCK decomposition. Beyond the above obvious examples, the following regular surfaces are known to admit an MCK decomposition: K3 surfaces, certain elliptic surfaces constructed by Schreieder [59] and Todorov surfaces of type $(0,9)$ or $(1,10)$. The case of $\mathrm{K} 3$ surfaces is seminal and is due to Beauville-Voisin [5] who establish the existence of a canonical 0 -cycle $o$ of degree 1 such that the modified small diagonal $\Gamma_{3}(S, o)$ vanishes. This was reinterpreted, via a direct computation, as saying that a $\mathrm{K} 3$ surface admits an MCK decomposition in [61, §8]. The case of the Schreieder surfaces is [42, Theorem 2], while the case of Todorov surfaces is the content of Theorem 1.4 to be proved.

Remark 4.3 (MCK decomposition on the image) Let $S$ be a smooth projective regular surface admitting a multiplicative Chow-Künneth decomposition (3). Let $f: S \rightarrow S^{\prime}$ be a surjective morphism to another smooth projective surface. Then $S^{\prime}$ must be regular and it admits a multiplicative Chow-Künneth decomposition. Indeed, by Proposition 4.1, we have a degree one 0-cycle $z$ on $S$ such that $\Gamma_{3}(S, z)=0$ in $\mathrm{CH}^{4}\left(S^{3}\right)$. It is easy to check that $(f, f, f)_{*}\left(\Gamma_{3}(S, z)\right)=\operatorname{deg}(f) \Gamma_{3}\left(S^{\prime}, f_{*}(z)\right)$. Again by Proposition $4.1, S^{\prime}$ has an MCK decomposition. Note that if $f: C \rightarrow C^{\prime}$ is a dominant morphism of curves, the same argument shows that an MCK decomposition for $C$ yields an MCK decomposition for $C^{\prime}$. 
Proposition 4.1 (iii) and (iv) give obstructions to the existence of multiplicative Chow-Künneth decompositions for regular surfaces. Moreover, we can use this to see the difference between (iii) and MCK decompositions:

Proposition 4.4 (MCK decomposition v.s. degeneration of intersection product). For any $d \geq 7$, a very general smooth surface of degree $d$ in $\mathbb{P}^{3}$ does not admit any multiplicative Chow-Künneth decomposition of the form (3). However, as a very general surface has Picard number 1, the conditions (iii) and (iv) in Proposition 4.1 are obviously satisfied with $z=\frac{1}{d} c_{1}(\mathcal{O}(1))^{2}$.

Proof Generalizing the octic surface example in [15, §1.4], O'Grady [52] constructed, for each integer $d$, a smooth surface $S$ of degree $d$ in $\mathbb{P}^{3}$ with

$$
\operatorname{dim} \operatorname{Im}\left(\mathrm{CH}^{1}(S) \otimes \mathrm{CH}^{1}(S) \rightarrow \mathrm{CH}^{2}(S)\right) \geq\left[\frac{d-1}{3}\right] .
$$

So by Proposition 4.1 such a surface $S$ does not have an MCK decomposition of the form (3) when $d$ is at least 7. By Proposition 4.1, the modified small diagonal $\Gamma_{3}\left(S, \frac{1}{d} c_{1}(\mathcal{O}(1))^{2}\right)$ does not vanish. However, since the cycle $\Gamma_{3}\left(S, \frac{1}{d} c_{1}(\mathcal{O}(1))^{2}\right)$ is defined universally for all smooth degree $d$ surfaces, its non-vanishing on one member, namely $S$, implies that it is non-trivial for a very general member by an argument using Hilbert schemes (cf. [72, Lemma 3.2]).

\subsection{On the tautological ring of powers of regular surfaces}

The following proposition due to Yin [74], which is the analogue of Proposition 3.5, gives yet another characterization of regular surfaces admitting an MCK decomposition.

Proposition 4.5 Let $S$ be a smooth projective complex surface. Let $\mathrm{R}^{*}\left(S^{m}\right) \subset \mathrm{CH}^{*}\left(S^{m}\right)$ denote the subring generated by pullbacks of divisors, Chern classes and diagonals $\Delta_{S}$. Assume that $S$ is regular and that $p_{g}:=\mathrm{H}^{0}\left(S, \Omega_{S}^{2}\right)>0$. Then $S$ admits an MCK decomposition of the form (3) if and only if the cycle class map induces injections

$$
\mathrm{R}^{i}\left(S^{m}\right) \hookrightarrow \mathrm{H}^{2 i}\left(S^{m}\right)
$$

for all $m \leq 2 b_{2, t r}(S)+1$ and all $i$. Here, $b_{2, t r}(S)$ is the dimension of the transcendental cohomology of $S$, i.e., the smallest Hodge substructure of $\mathrm{H}^{2}(S)$ whose complexification contains $\mathrm{H}^{0}\left(S, \Omega_{S}^{2}\right)$. Moreover, $S$ admits an MCK decomposition and is Kimura-O'Sullivan finitedimensional if and only if $\mathrm{R}^{i}\left(S^{m}\right) \rightarrow \mathrm{H}^{2 i}\left(S^{m}\right)$ is injective for all $m$ and all $i$.

Proof This is simply an application of Yin's theorem [74] (which works for any regular surface with $p_{g}>0$, see also [74, Remark 3.8(iii)]), via reinterpreting the vanishing of the modified small diagonal as providing an MCK decomposition. 


\section{Cubic hypersurfaces}

Smooth cubic fourfolds are the most well-known examples of Fano varieties of K3 type. They have the following Hodge diamond:

$\begin{array}{cccccccccc} & & & & 1 & & & & \\ & & & 0 & & 0 & & & \\ & & & & & 1 & & 0 & & \\ & & 0 & & 0 & & 0 & & 0 & \\ 0 & & 1 & & 21 & & 1 & & 0 . \\ & 0 & & 0 & & 0 & & 0 & \\ & & 0 & & 1 & & 0 & & \\ & & & 0 & & 0 & & & \\ & & & & 1 & & & & \\ & & & & & & & & & \end{array}$

Although our main aim is the case of smooth cubic hypersurfaces of dimension 4 , it turns out that the results of this section hold for smooth cubic hypersurfaces of any dimension $\geq 3$.

\subsection{Statement of the main result}

We start with the following general definition:

Definition 5.1 (Generically defined cycle classes and the Franchetta property) Let $\mathcal{X} \rightarrow B$ be a smooth projective morphism to a smooth quasi-projective complex variety $B$. For any fiber $X$ of $\mathcal{X} \rightarrow B$ over a closed point of $B$, we define

$$
\operatorname{GDCH}_{B}^{*}(X):=\operatorname{Im}\left(\mathrm{CH}^{*}(\mathcal{X}) \rightarrow \mathrm{CH}^{*}(X)\right),
$$

the image of the Gysin restriction map. The elements of $\mathrm{GDCH}_{B}^{*}(X)$, which by abuse we will denote $\operatorname{GDCH}^{*}(X)$ when $B$ is clear from the context, are called the generically defined cycles (with respect to the deformation family $B$ ) on $X$.

We say that $\mathcal{X} \rightarrow B$ has the Franchetta property if the restriction of the cycle class map

$$
\mathrm{GDCH}_{B}^{*}(X) \hookrightarrow \mathrm{CH}^{*}(X) \rightarrow \mathrm{H}^{*}(X)
$$

is injective for all fibers $X$ of $\mathcal{X} \rightarrow B$ (equivalently, for a very general fiber $X$; see [16, §2]).

This property is studied in $[51,55]$ for the universal family of $\mathrm{K} 3$ surfaces of low genus. This is extended to certain families of hyper-Kähler varieties in [16] (cf. also [6]), and most notably for the square of the Fano variety of lines on a smooth cubic fourfold. The aim of this section is to generalize the latter to the Fano variety of lines on a smooth cubic hypersurface of any dimension. Recall that if $X$ is a smooth cubic hypersurface in $\mathbb{P}^{n+1}$, then its Fano variety of lines, denoted $F$, is known to be connected smooth projective of dimension $2 n-4$.

Theorem 5.2 Let $B$ be the open subset of $\mathbb{P H}^{0}\left(\mathbb{P}^{n+1}, \mathcal{O}(3)\right)$ parameterizing smooth cubic hypersurfaces, and let $\mathcal{F} \rightarrow B$ be the universal family of their Fano varieties of lines. Then 
the families $\mathcal{F} \rightarrow B$ and $\mathcal{F} \times_{B} \mathcal{F} \rightarrow B$ have the Franchetta property. Moreover, if $F$ is the Fano variety of lines on a very general cubic hypersurface $X \subseteq \mathbb{P}^{n+1}$ of dimension $n>2$, then the cycle class map induces isomorphisms

$$
\operatorname{GDCH}^{*}(F) \stackrel{\sim}{\longrightarrow} \operatorname{Hdg}\left(\mathrm{H}^{*}(F)\right) \text { and } \operatorname{GDCH}^{*}(F \times F) \stackrel{\sim}{\longrightarrow} \operatorname{Hdg}\left(\mathrm{H}^{*}(F \times F)\right),
$$

where $\operatorname{Hdg}\left(\mathrm{H}^{*}(-)\right)$ denotes the subalgebra generated by Hodge classes in $\mathrm{H}^{*}(-)$.

A direct consequence of Theorem 5.2 is the following:

Corollary 5.3 Let $Y \subset \mathbb{P}^{n+1}$ be a smooth cubic hypersurface, and $F=F(Y)$ its Fano variety of lines. Then $F$ verifies the standard conjectures.

Proof First we note that the corollary follows from the motivic relation in [36]; we provide here, however, a new and self-contained proof. The statement is clearly true if $n \leq 2$, as then $F$ has dimension $\leq 0$. We thus assume that $n>2$. From Theorem 5.2, the cycle class map induces, for the very general $Y$, a surjection

$$
\operatorname{GDCH}^{*}(F \times F) \rightarrow \operatorname{Hdg}\left(\mathrm{H}^{*}(F \times F)\right) .
$$

Hence, for the very general $Y$ the Hodge class on $F \times F$ occurring in the standard conjecture of Lefschetz type for $F$ is algebraic and generically defined. A standard spread argument [72, Lemma 3.2] then allows to conclude the same is true for any smooth $Y$.

\subsection{MCK decomposition for cubic hypersurfaces}

Let $\mathcal{X} \rightarrow B$ be the universal family of smooth cubic hypersurfaces as above, and let $e: \mathcal{X} \rightarrow \mathbb{P}^{n+1}$ be the natural evaluation map. Set $H:=e^{*}\left(c_{1}(\mathcal{O}(1))\right) \in \mathrm{CH}^{1}(\mathcal{X})$, the relative hyperplane section class. Then

$$
\pi_{\mathcal{X}}^{2 i}:=\frac{1}{3} H^{n-i} \times_{B} H^{i} \quad \text { for } 2 i \neq n, \quad \text { and } \quad \pi_{\mathcal{X}}^{n}:=\Delta_{\mathcal{X} / B}-\sum_{2 i \neq n} \pi_{\mathcal{X}}^{2 i}
$$

defines a relative Chow-Künneth decomposition, in the sense that its specialization to any fiber $\mathcal{X}_{b}$ over a closed point $b \in B$ gives a Chow-Künneth decomposition of $\mathcal{X}_{b}$. A direct consequence of Theorem 5.2, together with the fact proved in Proposition 5.10 that the motive of a cubic hypersurface $X$ and of its symmetric square are (generically defined) direct factors of the motive of the Fano variety of lines $F$, is the following:

Theorem 5.4 [13] The relative Chow-Künneth decomposition (4) is fiberwise multiplicative. In particular, a smooth cubic hypersurface admits a multiplicative Chow-Künneth decomposition.

The above theorem is not stated in this form in [13]; it can, however, be deduced from [13, Corollary 3.3.9], as is explained in Sect. 4.6. In this section, we obtain Theorem 5.4 as a consequence of the stronger result Theorem 5.2, which will play a crucial role in our subsequent work [17] where we determine, among other things, the Chow motive of $F$ in terms of the Chow motive of $X$. Moreover, contrary to the approach employed in [13], our 
proof is independent of [36]; in fact, in [17] we strengthen and obtain a new independent and more conceptual proof of, the main result of [36]. The reader only interested in the case of cubic fourfolds can skip the proof of Theorem 5.2 given in Sect. 5.5 and rely on [16, Theorem 1.10].

\subsection{The Franchetta property for cubic hypersurfaces and their squares}

Before proceeding to the main proposition of this paragraph, we first introduce some notation. Given $H$ a pure Hodge structure of pure weight $w$, we denote $\mathrm{T}^{*} H$ the tensor algebra on $H, \operatorname{Sym}^{\circ} H$ the symmetric algebra on $H$ with the commutativity constraint imposed by the parity of the weight $w$ of $H$, and $\operatorname{hdg}(H)$ the dimension of the space of Hodge classes $\operatorname{Hdg}(H)$ in $H$.

Lemma 5.5 Let $X \subseteq \mathbb{P}^{n+1}$ be a very general cubic hypersurface of dimension $\neq 2$. Denote $H_{X}:=\mathrm{H}_{\text {prim }}^{n}(X)(1)$ the primitive cohomology of $X$ Tate twisted by 1 ; it is a pure Hodge structure of weight $n-2$. Then we have

$$
\begin{gathered}
\operatorname{hdg}\left(H_{X}\right)=\operatorname{hdg}\left(H_{X} \otimes \operatorname{Sym}^{2} H_{X}\right)=0, \\
\operatorname{hdg}\left(H_{X} \otimes H_{X}\right)=\operatorname{hdg}\left(\operatorname{Sym}^{2} H_{X}\right)=1, \\
\operatorname{hdg}\left(\operatorname{Sym}^{2} H_{X} \otimes \operatorname{Sym}^{2} H_{X}\right)=2 .
\end{gathered}
$$

Proof Given any very general hypersurface $X \subseteq \mathbb{P}^{n+1}$ with cohomology ring not generated by Hodge classes, a theorem of Deligne states that the Mumford-Tate group of $H_{X}$ coincides with the Zariski closure $G$ of the monodromy group of a general Lefschetz pencil acting on $\mathrm{H}^{n}(X)_{\text {prim }}$ and is the full orthogonal group if $n$ is even and the full symplectic group if $n$ is odd; see, e.g., [50]. Since the subspace of Hodge classes in tensor powers of $H_{X}$ is given by $G$-invariants, it follows from the invariant theory of the orthogonal and symplectic groups that the subalgebra of Hodge classes in the tensor algebra $\mathrm{T}^{*} H_{X}$ is generated by the quadratic form $q \in \mathrm{Sym}^{2} H_{X} \subset H_{X}^{\otimes 2}$ on $H_{X}$ in the case $n$ is even, and by the symplectic form, $q \in \operatorname{Sym}^{2} H_{X} \subset H_{X}^{\otimes 2}$ on $H_{X}$ in the case $n$ is odd. The lemma is then straightforward.

Proposition 5.6 Let $B$ be the open subset of $\mathbb{P H}^{0}\left(\mathbb{P}^{n+1}, \mathcal{O}(3)\right)$ parameterizing smooth cubic hypersurfaces, and let $\mathcal{X} \rightarrow B$ be the universal family. Then the families $\mathcal{X} \rightarrow B$ and $\mathcal{X} \times_{B} \mathcal{X} \rightarrow B$ have the Franchetta property. Moreover, when $n \neq 2$, for a very general fiber $X$, the cycle class map induces isomorphisms

$$
\mathrm{GDCH}^{*}(X) \stackrel{\sim}{\longrightarrow} \mathrm{Hdg}\left(\mathrm{H}^{*}(X)\right) \text { and } \operatorname{GDCH}^{*}(X \times X) \stackrel{\sim}{\longrightarrow} \operatorname{Hdg}\left(\mathrm{H}^{*}(X \times X)\right) .
$$

Proof First, let us determine generators for the rings $\operatorname{GDCH}^{*}(X)$ and $\operatorname{GDCH}^{*}(X \times X)$ (which were defined in Definition 5.1). It follows from [16, Proposition 5.2], since $\mathcal{X} \rightarrow B$ and $\mathcal{X} \times_{B} \mathcal{X} \rightarrow B$ are "stratified projective bundles" in the sense of loc. cit., that

$$
\mathrm{GDCH}^{*}(X)=\langle h\rangle \text { and } \operatorname{GDCH}^{*}(X \times X)=\left\langle p_{i}^{*} h, \Delta_{X}\right\rangle,
$$


where $h \in \mathrm{CH}^{1}(X)$ denotes the hyperplane section class and $p_{i}: X \times X \rightarrow X$ is the projection on the $i$ th factor.

It is then clear that the cycle class map $\operatorname{GDCH}^{*}(X) \hookrightarrow \mathrm{H}^{*}(X)$ is injective. The injectivity of the cycle class map $\operatorname{GDCH}^{*}(X \times X) \rightarrow \mathrm{H}^{*}(X \times X)$ is deduced easily from the following relation in $\mathrm{CH}^{n+1}(X \times X)$ :

$$
\Delta_{X} \cdot p_{1}^{*} h=\Delta_{X} \cdot p_{2}^{*} h=\frac{1}{3} \sum_{\substack{i+j=n+1 \\ i, j>0}} p_{1}^{*} h^{i} \cdot p_{2}^{*} h^{j} .
$$

To show (8), we consider the following Cartesian diagram whose excess normal bundle is $\mathcal{O}_{X}(3)$ :

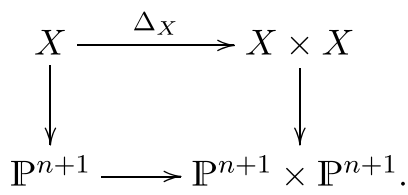

The excess intersection formula [21, Theorem 6.3] yields that

$$
\Delta_{X, *}\left(c_{1}\left(\mathcal{O}_{X}(3)\right)\right)=\left.\Delta_{\mathbb{P} n+1}\right|_{X \times X} .
$$

As $\Delta_{\mathbb{P}^{n+1}}=\sum_{i=0}^{n+1} h^{i} \times h^{n+1-i}$ in $\mathrm{CH}^{n+1}\left(\mathbb{P}^{n+1} \times \mathbb{P}^{n+1}\right)$, where with an abuse of notation $h$ denotes also the hyperplane section class in $\mathbb{P}^{n+1}$, we obtain that in $\mathrm{CH}(X \times X)$,

$$
\Delta_{X, *}(3 h)=\sum_{i=0}^{n+1} h^{i} \times h^{n+1-i},
$$

which is nothing else but (8).

Suppose now that $X$ is very general of dimension $\neq 2$. That $\operatorname{GDCH}^{*}(X) \rightarrow \operatorname{Hdg}\left(\mathrm{H}^{*}(X)\right)$ is surjective then follows directly from Lemma 5.5 and from the decomposition

$$
\mathrm{H}^{*}(X)=H_{X} \oplus \bigoplus_{i=0}^{n} \mathbb{Q}(-i),
$$

where each summand $\mathbb{Q}(-i)$ is spanned by $h^{i}$. By Lemma 5.5 again, in order to see that $\mathrm{GDCH}^{*}(X \times X) \rightarrow \operatorname{Hdg}\left(\mathrm{H}^{*}(X \times X)\right)$ is surjective, it suffices to note that the diagonal $\Delta_{X}$ accounts for the Hodge class appearing in $\operatorname{Sym}^{2} H_{X}$.

\subsection{Cubic hypersurfaces and their Fano varieties of lines}

If $X$ is a smooth cubic hypersurface in $\mathbb{P}^{n+1}$, we denote $F$ its Fano variety of lines. We first give a corrected proof of a result of Diaz [13, Proposition 3.2.6]. Let $g=-\left.c_{1}(\mathcal{E})\right|_{F}$ be the Plücker polarization and $c=\left.c_{2}(\mathcal{E})\right|_{F}$, where $\mathcal{E}$ is the tautological rank-2 bundle on $G:=\operatorname{Gr}(2, n+2)$. 
Lemma 5.7 In $\mathrm{CH}^{n-1}(F), g^{n-1}$ is a linear combination of $g^{n-3} c, g^{n-5} c^{2}, \ldots$.

Proof In the case where $n \leq 4$ the result is already contained in [71]. We thus assume that $n \geq 5$. Recall that $G:=\operatorname{Gr}(2, n+2)$ denotes the Grassmannian variety parameterizing projective lines in $\mathbb{P}^{n+1}$; as such, $\imath: F \hookrightarrow G$ is a codimension 4 closed subscheme. As in [13], for dimension reasons there exists a nonzero polynomial $P(x, y) \in \mathbb{Q}[x, y]$ homogeneous of weighted degree $n-1$, where $x$ has degree 1 and $y$ has degree 2, such that the push-forward of $P(g, c) \in \mathrm{CH}^{n-1}(F)$ to $\mathrm{CH}^{n+3}(G)$ is zero, i.e.,

$$
P(g, c) \cdot[F]=0 \text { in } \mathrm{CH}^{n+3}(G) .
$$

Let us define $\mathrm{R}^{*}(F):=\imath^{*} \mathrm{CH}^{*}(G) \subset \mathrm{CH}^{*}(F)$. Since homological and numerical equivalence agree on $F$ (Proposition 5.11), the push-forward map $l_{*}: \mathrm{R}^{*}(F) \rightarrow \mathrm{CH}^{*+4}(G)$ is injective. It follows that $P(g, c)$ gives a linear relation between $g^{n-1}, g^{n-3} c, \ldots$ in $\mathrm{CH}^{n-1}(F)$. To prove the lemma, it suffices to show that the polynomial $P(x, y)$ is not divisible by $y$.

We know that $[F]=18 g^{2} c+9 c^{2}$ in $\mathrm{CH}^{4}(G)$ [21, Example 14.7.13]. We also know that the ideal of relations in $\mathrm{CH}^{*}(G)$ is generated by the following two polynomials

$$
\begin{aligned}
& R_{n+1}(g, c)=g^{n+1}-\left(\begin{array}{c}
n \\
1
\end{array}\right) g^{n-1} c+\left(\begin{array}{c}
n-1 \\
2
\end{array}\right) g^{n-3} c^{2}-\cdots \\
& R_{n+2}(g, c)=g^{n+2}-\left(\begin{array}{c}
n+1 \\
1
\end{array}\right) g^{n} c+\left(\begin{array}{l}
n \\
2
\end{array}\right) g^{n-2} c^{2}-\cdots
\end{aligned}
$$

(In the proof of [13, Proposition 3.2.6], it is wrongly claimed that the ideal of relations in $\mathrm{CH}^{*}(G)$ is generated by two homogeneous polynomials of degree $n$ and $n+1$, respectively.) It thus follows from (9) that there is equality of polynomials (of degree $n+3$ )

$$
P(x, y) \cdot\left(2 x^{2} y+y^{2}\right)=\left(m_{1} x^{2}+m_{2} y\right) R_{n+1}(x, y)+m_{3} x R_{n+2}(x, y),
$$

for some $m_{j} \in \mathbb{Q}$. Let us assume, by contradiction, that the polynomial $P(x, y)$ is divisible by $y$, i.e., that we can write

$$
P(x, y)=p_{1} x^{n-3} y+p_{2} x^{n-5} y^{2}+\cdots+p_{m} x^{n-1-2 m} y^{m},
$$

where $p_{j} \in \mathbb{Q}$ and $m:=\left\lfloor\frac{n-1}{2}\right\rfloor \geq 2$. Comparing the $x^{n+3}$ terms on both sides of (10), we find that $m_{3}=-m_{1}$. Comparing the $x^{n+1} y$ terms on both sides of (10), we find that $m_{2}=-m_{1}$. The polynomial $P(x, y)$ being nonzero, we may assume that $m_{1}$ is nonzero. Up to rescaling $P(x, y)$, we may thus assume that $m_{1}=1$. Let us now develop equality (10) with $m_{1}=1$ and $m_{2}=m_{3}=-1$. The right-hand side of (10) can be written

$$
\sum_{j=2}^{m+2} a_{j} x^{n+3-2 j} y^{j}
$$

where the coefficient $a_{j}$ is

$$
a_{j}=(-1)^{j+1}\left(\left(\begin{array}{c}
n+2-j \\
j
\end{array}\right)-\left(\begin{array}{c}
n+1-j \\
j
\end{array}\right)-\left(\begin{array}{c}
n+2-j \\
j-1
\end{array}\right)\right)=(-1)^{j}\left(\begin{array}{c}
n+1-j \\
j-1
\end{array}\right) .
$$

The equality of polynomials (10) implies the equalities of coefficients 


$$
\begin{aligned}
2 p_{1} & =a_{2}, \\
2 p_{2}+p_{1} & =a_{3}, \\
2 p_{3}+p_{2} & =a_{4}, \\
& \vdots \\
2 p_{m}+p_{m-1} & =a_{m+1}, \\
p_{m} & =a_{m+2} .
\end{aligned}
$$

Note that the $a_{j}$ are integers. On the other hand, we observe that $p_{2}$ is not an integer. (Indeed, from (11) we find that $p_{1}=\frac{n-1}{2}$, and $p_{2}=\frac{1}{2}\left(a_{3}-p_{1}\right)=-\frac{1}{4}\left(n^{2}-4 n+5\right)$, which is not an integer.) It follows inductively that $p_{j}$ is not an integer for any $j \geq 2$ (indeed, from (11) we find that $2 p_{j}=a_{j+1}-p_{j-1}$ is not integer). But this contradicts the last line of (11), and so $P(x, y)$ is not divisible by $y$.

We now generalize to arbitrary dimension a result of Beauville-Donagi [2, Proposition 6] for cubic fourfolds.

Proposition 5.8 Let $X$ be a very general cubic hypersurface of dimension $n>2$. Let $F$ be its Fano variety of lines and $g \in \mathrm{CH}^{1}(F)$ be the first Chern class of the Plücker polarization. Let $p: P \rightarrow F$ be the universal $\mathbb{P}^{1}$-bundle and $q: P \rightarrow X$ be the natural evaluation morphism. Then

(i) the morphism of Hodge structures $p_{*} q^{*}: \mathrm{H}^{n}(X, \mathbb{Q})(1) \rightarrow \mathrm{H}^{n-2}(F, \mathbb{Q})$ is injective, and it is an isomorphism if $n$ is odd;

(ii) there exists a nonzero rational number $\lambda$ such that for any $\alpha, \alpha^{\prime} \in \mathrm{H}_{\text {prim }}^{n}(X, \mathbb{Q})$, we have

$$
\lambda\left\langle\alpha, \alpha^{\prime}\right\rangle_{X}=\left\langle p_{*} q^{*}(\alpha), p_{*} q^{*}\left(\alpha^{\prime}\right)\right\rangle_{F},
$$

where $\langle-,-\rangle_{X}$ is the intersection pairing on $X$, while $\left\langle\beta, \beta^{\prime}\right\rangle_{F}:=\int_{F} \beta \cdot \beta^{\prime} \cdot g^{n-2}$ for any $\beta, \beta^{\prime} \in \mathrm{H}^{n-2}(F, \mathbb{Q})$;

(iii) The morphism $p_{*} q^{*}$ respects the primitive parts: $p_{*} q^{*}\left(\mathrm{H}_{\text {prim }}^{n}(X, \mathbb{Q})(1)\right) \subset \mathrm{H}_{\text {prim }}^{n-2}(F, \mathbb{Q})$. It is an equality if $n$ is odd or divisible by 4 ; when $4 \mid n-2$, the complement is onedimensional, generated by $c^{\frac{n-2}{4}}$;

(iv) The morphism $p_{*} q^{*}$ induces an isomorphism of transcendental cohomology groups:

$$
p_{*} q^{*}: \mathrm{H}_{\mathrm{tr}}^{n}(X, \mathbb{Q})(1) \stackrel{\sim}{\longrightarrow} \mathrm{H}_{\mathrm{tr}}^{n-2}(F, \mathbb{Q}) .
$$

(v) When $n$ is even, $\mathrm{H}^{n-2}(F, \mathbb{Q}) \cong \mathrm{H}_{\text {prim }}^{n}(X, \mathbb{Q})(1) \oplus \mathbb{Q}\left(-\frac{n-2}{2}\right)^{\oplus\left\lfloor\frac{n+2}{4}\right\rfloor}$, where the isomorphism is given by $p_{*} q^{*}$ on the summand $\mathrm{H}_{\text {prim }}^{n}(X, \mathbb{Q})(1)$, and by $g^{\frac{n}{2}+1-2 i} c^{i-1}$ on the ith copy of $\mathbb{Q}\left(-\frac{n-2}{2}\right)$, for $1 \leq i \leq\left\lfloor\frac{n+2}{4}\right\rfloor$.

Proof We adapt the argument of [2]. For (i), by Shimada [62, 63] (see [27, Theorem 4] for an alternative proof), the cylinder map $q_{*} p^{*}: \mathrm{H}^{3 n-6}(F, \mathbb{Q}) \rightarrow \mathrm{H}^{n}(X, \mathbb{Q})$ is surjective. 
Hence, the transposed morphism $p_{*} q^{*}: \mathrm{H}^{n}(X, \mathbb{Q}) \rightarrow \mathrm{H}^{n-2}(F, \mathbb{Q})$ is injective. When $n$ is odd, we know that $\operatorname{dim} \mathrm{H}^{n}(X, \mathbb{Q})=\operatorname{dim} \mathrm{H}^{n-2}(F, \mathbb{Q})$ (see, for example, [22, Theorem 6.1]); hence, $p_{*} q^{*}$ must be an isomorphism.

For (ii), given $\alpha, \alpha^{\prime} \in \mathrm{H}_{\text {prim }}^{n}(X, \mathbb{Q})$, using the projective bundle formula, there exist $\beta_{1}, \beta_{1}^{\prime} \in \mathrm{H}^{n}(F, \mathbb{Q})$ and $\beta_{2}, \beta_{2}^{\prime} \in \mathrm{H}^{n-2}(F, \mathbb{Q})$ such that

$$
q^{*}(\alpha)=p^{*}\left(\beta_{2}\right) \cdot \xi-p^{*}\left(\beta_{1}\right) \quad \text { and } \quad q^{*}\left(\alpha^{\prime}\right)=p^{*}\left(\beta_{2}^{\prime}\right) \cdot \xi-p^{*}\left(\beta_{1}^{\prime}\right),
$$

where $\xi:=c_{1}\left(\mathcal{O}_{p}(1)\right)=q^{*}(H)$ with $H$ denoting the hyperplane section class on $X$. Applying $p_{*}$ to (12), we find that

$$
p_{*} q^{*}(\alpha)=\beta_{2} .
$$

Denote $\mathcal{E}$ the tautological rank-2 bundle on $\operatorname{Gr}\left(\mathbb{P}^{1}, \mathbb{P}^{n+1}\right)$ and set $g:=-\left.c_{1}(\mathcal{E})\right|_{F}$ to be the Plücker polarization and $c=\left.c_{2}(\mathcal{E})\right|_{F}$. Multiplying (12) by $\xi=q^{*}(H)$ and using the equality $\xi^{2}=p^{*}(g) \xi-p^{*}(c)$, we obtain

$$
0=q^{*}(\alpha \cdot H)=p^{*}\left(\beta_{2}\right) \cdot \xi^{2}-p^{*}\left(\beta_{1}\right) \cdot \xi=p^{*}\left(\beta_{2} \cdot g\right) \cdot \xi-p^{*}\left(\beta_{2} \cdot c\right)-p^{*}\left(\beta_{1}\right) \cdot \xi .
$$

Therefore,

$$
\beta_{1}=\beta_{2} \cdot g \text { and } \beta_{2} \cdot c=0,
$$

and similarly for $\beta_{1}^{\prime}$ and $\beta_{2}^{\prime}$. The identities (12) thus become

$$
q^{*}(\alpha)=p^{*}\left(\beta_{2}\right) \cdot \xi-p^{*}\left(\beta_{2} \cdot g\right) \quad \text { and } \quad q^{*}\left(\alpha^{\prime}\right)=p^{*}\left(\beta_{2}^{\prime}\right) \cdot \xi-p^{*}\left(\beta_{2}^{\prime} \cdot g\right) .
$$

Taking the product of the equations in (13), using again $\xi^{2}=p^{*}(g) \xi-p^{*}(c)$ and $\beta_{2} \cdot c=\beta_{2}^{\prime} \cdot c=0$, one obtains

$$
q^{*}\left(\alpha \cdot \alpha^{\prime}\right)=p^{*}\left(\beta_{2} \cdot \beta_{2}^{\prime} \cdot g^{2}\right)-p^{*}\left(\beta_{2} \cdot \beta_{2}^{\prime} \cdot g\right) \cdot \xi .
$$

Multiplying with $p^{*}\left(g^{n-3}\right)$ and then applying $q_{*}$, we find

$$
\alpha \cdot \alpha^{\prime} \cdot q_{*} p^{*}\left(g^{n-3}\right)=-q_{*} p^{*}\left(\beta_{2} \cdot \beta_{2}^{\prime} \cdot g^{n-2}\right) .
$$

Since $q_{*} p^{*}\left(g^{n-3}\right)$ is a nonzero multiple of the fundamental class of $X$, taking the degree of both sides yields that

$$
\lambda\left\langle\alpha, \alpha^{\prime}\right\rangle_{X}=\operatorname{deg}\left(\beta_{2} \cdot \beta_{2}^{\prime} \cdot g^{n-2}\right),
$$

for some $\lambda \neq 0$. This is nothing else but the desired formula in (ii).

For (iii), let us first show that $\beta_{2}=p_{*} q^{*}(\alpha)$ is in the primitive part, i.e., $\beta_{2} \cdot g^{n-1}=0$. However, $g^{n-1}$ is a linear combination of $g^{n-3} c, g^{n-5} c^{2}, \ldots$ (see, for example, Lemma 5.7); hence, the desired vanishing follows from the vanishing $\beta_{2} \cdot c=0$. The inclusion is proved. When $n$ is odd or divisible by 4 , by [22, Theorem 6.1], $\left.\operatorname{dim} \mathrm{H}_{\text {prim }}^{n}(X, \mathbb{Q})(1)\right)=\operatorname{dim} \mathrm{H}_{\text {prim }}^{n-2}(F, \mathbb{Q})$; hence, the inclusion must be an equality. When $4 \mid n-2$, again by [22, Theorem 6.1], the complement is one-dimensional. It suffices to see that $c^{\frac{n-2}{4}}$ is not in $p_{*} q^{*}\left(\mathrm{H}_{\text {prim }}^{n}(X, \mathbb{Q})(1)\right)$. Indeed, one checks easily that $c^{\frac{n-2}{4}}$ is orthogonal to $p_{*} q^{*}\left(\mathrm{H}_{\text {prim }}^{n}(X, \mathbb{Q})(1)\right)$ with respect to $\langle-,-\rangle_{F}$. 
For $(i v)$, it is clear that $p_{*} q^{*}$ induces a morphism between $\mathrm{H}_{\mathrm{tr}}^{n}(X, \mathbb{Q})$ and $\mathrm{H}_{\mathrm{tr}}^{n-2}(F, \mathbb{Q})$, which is injective by $(i)$. To see its surjectivity, it suffices to observe that $\mathrm{H}_{\mathrm{tr}}^{n}(X, \mathbb{Q})$ and $\mathrm{H}_{\mathrm{tr}}^{n-2}(F, \mathbb{Q})$ have the same dimension (see, for example, [22, Theorem 6.1]).

For $(v)$, by the dimension count in [22, Theorem 6.1], it suffices to show that $\mathrm{H}^{n-2}(F, \mathbb{Q})$ is spanned by $p_{*} q^{*}\left(\mathrm{H}_{\text {prim }}^{n}(X, \mathbb{Q})(1)\right)$ and $g^{\frac{n}{2}+1-2 i} c^{i-1}$, for $1 \leq i \leq\left\lfloor\frac{n+2}{4}\right\rfloor$, but this follows from the description of $\mathrm{H}_{\text {prim }}^{n-2}(F, \mathbb{Q})$ in $(i i i)$ and the fact that $\mathrm{H}^{n-4}(F, \mathbb{Q})$ is of Tate type and is generated by $g^{\frac{n}{2}-2 i} c^{i-1}$, for $1 \leq i \leq\left\lfloor\frac{n-2}{4}\right\rfloor$.

Corollary 5.9 The composition of the following chain of isomorphisms is a nonzero multiple of the identity map:

$$
\mathrm{H}_{\text {prim }}^{n}(X, \mathbb{Q}) \stackrel{p_{*} q^{*}}{\longrightarrow} \mathrm{H}_{\text {prim }}^{n-2}(F, \mathbb{Q}) \stackrel{\cdot g^{n-2}}{\longrightarrow} \mathrm{H}_{\text {prim }}^{3 n-6}(F, \mathbb{Q}) \stackrel{q_{*} p^{*}}{\longrightarrow} \mathrm{H}_{\text {prim }}^{n}(X, \mathbb{Q}) .
$$

Proof This is actually a general fact in linear algebra. Let $\Lambda_{1}, \Lambda_{2}$ be two $\mathbb{Q}$-quadratic spaces. If there is a linear isomorphism $\phi: \Lambda_{1} \stackrel{\cong}{\rightarrow} \Lambda_{2}$ and a nonzero rational number $\lambda$, such that $\lambda\langle x, y\rangle_{\Lambda_{1}}=\langle\phi(x), \phi(y)\rangle_{\Lambda_{2}}$, then the composition of the following chain of isomorphisms is $\lambda \cdot \mathrm{id}$.

$$
\begin{aligned}
& \Lambda_{1} \stackrel{\phi}{\longrightarrow} \Lambda_{2} \stackrel{\cong}{\longrightarrow} \Lambda_{2}^{\vee} \stackrel{{ }^{t} \phi}{\longrightarrow} \Lambda_{1}^{\vee} \stackrel{\cong}{\longrightarrow} \Lambda_{1} \\
& x \longmapsto \\
& \longrightarrow \phi(x) \longmapsto \\
&
\end{aligned}
$$

Thanks to Proposition 5.8, we can apply this general fact to the case when $\Lambda_{1}:=\mathrm{H}_{\text {prim }}^{n}(X, \mathbb{Q}), \Lambda_{2}:=\mathrm{H}_{\text {prim }}^{n-2}(F, \mathbb{Q})$, and $\phi:=p_{*} q^{*}$.

We denote $\mathcal{F} \rightarrow B$ the relative Fano variety of lines for the universal family $\mathcal{X} \rightarrow B$ of cubic hypersurfaces. The relative Chow motives of $\mathcal{X}$ and $\mathcal{F}$ over $B$ can be related thanks to the following construction, performed for smooth cubic hypersurfaces of any dimension, due to Galkin-Shinder [22] and Voisin [73]. Let $X$ be a smooth cubic hypersurface, and let $F$ be its Fano variety of lines. Galkin-Shinder [22, Proof of Theorem 5.1] constructed a birational map $\phi: X^{[2]} \rightarrow P_{X}$ from the Hilbert scheme of length-2 subschemes $X^{[2]}$ of $X$, where $P_{X}:=\mathbb{P}\left(\left.T_{\mathbb{P}^{n+1}}\right|_{X}\right)$ is some $\mathbb{P}^{n}$-bundle over $X$. Moreover, Voisin [73, Proposition 2.9] constructed an explicit resolution of indeterminacies 


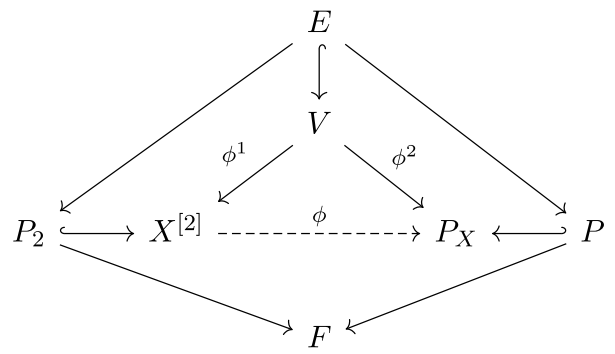

with the property that the morphism $\phi^{2}: V \rightarrow P_{X}$ is a blow-up along a smooth center $P \subset P_{X}$ of codimension 3, where $P$ is the universal $\mathbb{P}^{1}$-bundle over $F$, and the morphism $\phi^{1}: V \rightarrow X^{[2]}$ is a blow-up along a smooth center $P_{2} \subset X^{[2]}$ of codimension 2 , where $P_{2}$ is the relative symmetric square of $P \rightarrow F$, and thus has the structure of a $\mathbb{P}^{2}$-bundle over $F$. Since the above construction of Galkin-Shinder and Voisin can be performed familywise, we obtain thanks to the projective bundle formula and the blow-up formula for Chow motives an isomorphism of relative motives over $B$

$$
\begin{aligned}
\mathfrak{h}(\mathcal{V}) & \simeq \bigoplus_{i=0}^{n} \mathfrak{h}(\mathcal{X})(-i) \oplus \mathfrak{h}(\mathcal{F})(-3) \oplus \mathfrak{h}(\mathcal{F})(-2)^{\oplus 2} \oplus \mathfrak{h}(\mathcal{F})(-1) \\
& \simeq \mathfrak{h}\left(\mathcal{X}^{[2]}\right) \oplus \mathfrak{h}(\mathcal{F})(-3) \oplus \mathfrak{h}(\mathcal{F})(-2) \oplus \mathfrak{h}(\mathcal{F})(-1)
\end{aligned}
$$

From the above isomorphism, we can derive the following proposition that will be used in our new proof of Theorem 5.4. It will also be used in the proof of Theorem 5.2 to establish the Franchetta property of the family $\mathcal{F} \rightarrow B$.

Proposition 5.10 Let $\mathcal{X} \rightarrow B$ denote the universal smooth cubic hypersurface in $\mathbb{P}^{n+1}$ with $n>2, \mathcal{X}^{(2)} \rightarrow B$ its relative symmetric square, and let $\mathcal{F} \rightarrow B$ denote the universal Fano variety of lines in a cubic hypersurface. There exist relative morphisms of Chow motives over $B$

$$
\Phi: \mathfrak{h}(\mathcal{X}) \longrightarrow \mathfrak{h}(\mathcal{F})(1) \oplus \bigoplus \mathbb{1}_{B}(*)
$$

and

$$
\Psi: \mathfrak{h}\left(\mathcal{X}^{(2)}\right) \longrightarrow \bigoplus \mathfrak{h}(\mathcal{F})(*) \oplus \bigoplus \mathbb{1}_{B}(*)
$$

which are fiberwise split injective. Here, $\bigoplus \mathbb{1}_{B}(*)$ means a direct sum of relative Lefschetz motives and $\bigoplus \mathfrak{h}(\mathcal{F})(*)$ means a direct sum of Tate twists of $\mathfrak{h}(\mathcal{F})$.

Proof Let $\mathcal{P} \subset \mathcal{F} \times_{B} \mathcal{X}$ be the relative universal line, also seen as a relative morphism of motives

$$
\mathcal{P}: \mathfrak{h}(\mathcal{X}) \longrightarrow \mathfrak{h}(\mathcal{F})(1)
$$

Let $\pi_{\mathcal{X}}^{j}$ be the relative Chow-Künneth decomposition (4), and let $\mathfrak{h}^{n, \text { prim }}(\mathcal{X})$ denote the motive defined by the projector 
$\pi_{\mathcal{X}}^{n \text {,prim }}=\pi_{\mathcal{X}}^{n}$ in case $\mathrm{n}$ is odd, and $\pi_{\mathcal{X}}^{n \text {,prim }}=\pi_{\mathcal{X}}^{n}-\frac{1}{3} H^{n / 2} \times_{B} H^{n / 2} \quad$ in case $\mathrm{n}$ is even,

where $H \in \mathrm{CH}^{1}(\mathcal{X})$ denotes the relative hyperplane section. Corollary 5.9 implies that the composition

$$
\mathfrak{h}_{\text {prim }}^{n}(\mathcal{X}) \hookrightarrow \mathfrak{h}(\mathcal{X}) \stackrel{\mathcal{P}}{\longrightarrow} \mathfrak{h}(\mathcal{F})(1) \stackrel{\cdot g^{n-2}}{\longrightarrow} \mathfrak{h}(\mathcal{F})(-1) \stackrel{{ }^{t} \mathcal{P}}{\longrightarrow} \mathfrak{h}(X) \rightarrow \mathfrak{h}_{\text {prim }}^{n}(\mathcal{X})
$$

where $g$ denotes the relative Plücker polarization, induces fiberwise on $\mathrm{H}_{\text {prim }}^{n}(X)$ a nonzero multiple of the identity. The Franchetta property for $\mathcal{X} \times_{B} \mathcal{X} \rightarrow B$, proved in Proposition 5.6, shows that $\pi_{\mathcal{X}}^{n \text {, prim }}{ }^{t} \mathcal{P} \circ\left(\cdot g^{n-2}\right) \circ \mathcal{P} \circ \pi_{\mathcal{X}}^{n \text {, prim }}$ is equal to a nonzero multiple of $\pi_{\mathcal{X}}^{n \text {, prim }}$. It follows that $\mathcal{P} \circ \pi_{\mathcal{X}}^{n \text {, prim }}: \mathfrak{h}_{\text {prim }}^{n}(\mathcal{X}) \rightarrow \mathfrak{h}(\mathcal{F})(1)$ is fiberwise split injective, from which we deduce the existence of a morphism $\Phi$ as in the statement of the proposition.

Let us now turn to the existence of the morphism $\Psi$ as in the statement. First, recall that for any smooth projective variety $X$, the Hilbert scheme $X^{[2]}$ is the blow-up of the symmetric square $X^{(2)}$ along the diagonal. Therefore, in our relative situation, we have an isomorphism of relative Chow motives

$$
\mathfrak{h}\left(\mathcal{X}^{[2]}\right) \simeq \mathfrak{h}\left(\mathcal{X}^{(2)}\right) \oplus \bigoplus_{i=1}^{n-1} \mathfrak{h}(\mathcal{X})(-i)
$$

Combining the above isomorphism with the isomorphism (16) and with the morphism of relative motives $\Phi$ constructed above, we obtain the desired, fiberwise split injective, morphism $\Psi$.

Let us also mention the following direct consequence of the isomorphism (16).

Proposition 5.11 Let $F$ be the Fano variety of lines on a smooth cubic hypersurface $X$. Then homological and numerical equivalence agree on $F$.

Proof The statement is clearly true if $\operatorname{dim} X \leq 2$ since then $\operatorname{dim} F \leq 0$. We thus assume that $\operatorname{dim} X>2$. By specializing the relative isomorphism (16) to the fiber corresponding to $X$, we obtain an isomorphism of motives

$$
M \oplus P_{1} \oplus P_{2} \simeq N \oplus P_{1},
$$

where $\quad M=\bigoplus_{i=0}^{n} \mathfrak{h}(X)(-i), N=\mathfrak{h}\left(X^{[2]}\right), P_{1}=\mathfrak{h}(F)(-3) \oplus \mathfrak{h}(F)(-2) \oplus \mathfrak{h}(F)(-1) \quad$ and $\quad P_{2}=\mathfrak{h}(F)(-2)$. Sincethe standard conjectureshold for smooth hypersurfaces, and since $X^{[2]}$ is theblow-up of $X^{(2)}$ along the diagonal, homological and numerical equivalence agree on $M$ and also on $N$. Now, comparing the dimension of the kernels of $\mathrm{CH}^{*}\left(M \oplus P_{1} \oplus P_{2}\right) / \mathrm{hom} \rightarrow \mathrm{CH}^{*}\left(M \oplus P_{1} \oplus P_{2}\right) /$ num and of $\mathrm{CH}^{*}\left(N \oplus P_{1}\right) /$ hom $\rightarrow \mathrm{CH}^{*}\left(N \oplus P_{1}\right) /$ num, we find that $\operatorname{ker}\left(\mathrm{CH}^{*}\left(P_{2}\right) /\right.$ hom $\rightarrow$ $\mathrm{CH}^{*}\left(P_{2}\right) /$ num $)=0$, i.e., that homological and numerical equivalence agree on $F$.

\subsection{Proof of Theorem 5.2}

The case of cubic fourfolds is one of the main results of [16]. The point we want to make here is that, with the input of the isomorphism of Galkin-Shinder-Voisin (16), the original proof in [16] actually works in any dimension with minor adaptation. Let $F$ be the Fano 
variety of lines on a smooth cubic hypersurface $X$. As before, let us denote $\mathrm{GDCH}^{*}(F)$ and $\mathrm{GDCH}^{*}(F \times F)$ the subrings of $\mathrm{CH}^{*}(F)$ and $\mathrm{CH}^{*}(F \times F)$, respectively, generated by cycles that are restrictions of cycles on the universal families $\mathcal{F} \rightarrow B$ and $\mathcal{F} \times{ }_{B} \mathcal{F} \rightarrow B$.

Our previous results [16, Lemma 3.1 and Proposition 6.3] work without change and give us generators for $\operatorname{GDCH}^{*}(F)$ and $\operatorname{GDCH}^{*}(F \times F)$. More precisely, it is shown that these generically defined cycles are "tautological":

$$
\mathrm{GDCH}^{*}(F)=\mathrm{R}^{*}(F):=\langle g, c\rangle \text { and } \operatorname{GDCH}^{*}(F \times F)=\mathrm{R}^{*}(F \times F):=\left\langle p_{i}^{*} g, p_{j}^{*} c, \Delta_{F}, I\right\rangle,
$$

where $\langle-\rangle$ means the generated subalgebra, $p_{i}: F \times F \rightarrow F$ is the projection on the $i$ th factor, $g=-\left.c_{1}(\mathcal{E})\right|_{F}$ is the Plücker polarization, $c=\left.c_{2}(\mathcal{E})\right|_{F}, \mathcal{E}$ is the tautological rank-2 bundle on $G:=\operatorname{Gr}(2, n+2)$ and $I=\left\{\left(\ell_{1}, \ell_{2}\right) \in F \times F: \ell_{1} \cap \ell_{2} \neq \emptyset\right\}$ is the incidence correspondence.

Let us first show the Franchetta property for $\mathcal{F} \rightarrow B$. Since $\operatorname{GDCH}^{*}(X), \operatorname{GDCH}^{*}(X \times X)$ and $\mathrm{GDCH}^{*}(F)$ are finitely generated, we may use the isomorphism (16) and proceed as in the proof of Proposition 5.11; thanks to Proposition 5.6, rational and numerical equivalence agree on $\operatorname{GDCH}^{*}(X)$ and on $\operatorname{GDCH}^{*}(X \times X)$; therefore, the argument in Proposition 5.11 shows that rational and numerical equivalence agree on $\operatorname{GDCH}^{*}(F)$, i.e., that the family $\mathcal{F} \rightarrow B$ has the Franchetta property.

As a consequence, $\mathrm{R}^{*}(F)$ is a Gorenstein algebra ${ }^{1}$ of socle degree $2 n-4$. We can therefore bound the dimensions of the $\mathrm{R}^{i}(F)$ (we will see shortly that the following are actually equalities):

$$
\operatorname{dim} \mathrm{R}^{i}(F) \leq r_{i}:= \begin{cases}\left\lfloor\frac{i+2}{2}\right\rfloor, & \text { if } i \leq n-2 \\ \left\lfloor\frac{2 n-2-i}{2}\right\rfloor, & \text { if } i>n-2\end{cases}
$$

(We note that for $i=n-1$, the above dimension estimate takes the more precise form of Lemma 5.7).

In the system of generators (17) for $\mathrm{R}^{*}(F \times F)$, we first observe that the generator $\Delta_{F}$ is redundant, thanks to the following Voisin's relation [71] which holds in any dimension with the same proof:

$$
I^{2}=\lambda \Delta_{F}+I \cdot \Gamma_{1}\left(g_{1}, g_{2}\right)+\Gamma_{2}\left(g_{1}, g_{2}, c_{1}, c_{2}\right),
$$

where $\Gamma_{1}, \Gamma_{2}$ are polynomials and $\lambda$ is a nonzero rational number.

Next, we have the following relations, where $\alpha, \beta$ are nonzero rational numbers and $P, Q, R$ are polynomials. Note that their proof, originally for cubic fourfolds, works without change in any dimension.

(i) In [61, Proposition 17.5], one finds

$$
\Delta_{F} \cdot I=\alpha c_{1} \cdot \Delta_{F}-\beta g_{1}^{2} \cdot \Delta_{F} .
$$

(ii) In [61, Lemma 17.6], there is a polynomial $P$ such that

$$
\begin{aligned}
& c_{1} \cdot I=P\left(g_{1}, g_{2}, c_{1}, c_{2}\right) ; \\
& c_{2} \cdot I=P\left(g_{2}, g_{1}, c_{2}, c_{1}\right) .
\end{aligned}
$$

\footnotetext{
${ }^{1}$ A finite-dimensional graded algebra $A^{*}=\bigoplus_{i=0}^{d} A^{i}$ is called Gorenstein of socle degree $d$, if $A^{d}$ is onedimensional and the multiplication $A^{i} \times A^{d-i} \rightarrow A^{d}$ is a perfect pairing for all $0 \leq i \leq d$.
} 
(iii) In [16, Appendix], we proved there exists a polynomial $Q$ such that

$$
\Delta_{F, *}(g)+R\left(g_{1}, g_{2}\right) \cdot I=Q\left(g_{1}, g_{2}, c_{1}, c_{2}\right) .
$$

By Voisin's relation (19), we can eliminate the usage of $\Delta_{F}$ in previous relations (i) and (iii) to obtain the following relations, where $P, Q, R$ are some polynomials and $\mu$ is a nonzero rational number.

(i') $\quad I^{3}=\mu I \cdot g_{1}^{n-2} g_{2}^{n-2}+R\left(g_{1}, g_{2}, c_{1}, c_{2}\right)$;

(iii') $I^{2} \cdot g_{1}=I \cdot P\left(g_{1}, g_{2}\right)+Q\left(g_{1}, g_{2}, c_{1}, c_{2}\right)$.

Using Lemma 5.7, and relations (19), $\left(i^{\prime}\right),(i i)$ and (iii'), we get a system of linear generators for $\mathrm{R}^{*}(F \times F)$, namely

$$
\mathrm{R}^{*}(F \times F)=\mathrm{R}^{*}(F) \bigotimes \mathrm{R}^{*}(F)+I \cdot\left(\operatorname{Span}_{\mathbb{Q}}\left\{1, g, \ldots, g^{n-2}\right\} \otimes \operatorname{Span}_{\mathbb{Q}}\left\{1, g, \ldots, g^{n-2}\right\}\right)+\mathbb{Q} \cdot I^{2} .
$$

Combining with (18), we can bound the dimensions of $\mathrm{R}^{*}(F \times F)$ as follows, where the numbers $r_{i}$ are defined in (18). We will see that these are equalities in the end.

$$
\operatorname{dim} \mathrm{R}^{k}(F \times F) \leq \begin{cases}\sum_{i=0}^{k} r_{i} r_{k-i}, & \text { if } 0 \leq k<n-2 ; \\ \sum_{i=0}^{k} r_{i} r_{k-i}+(k-(n-2)+1), & \text { if } n-2 \leq k<2 n-4 ; \\ \sum_{i=0}^{2 n-4} r_{i} r_{k-i}+(n-1)+1, & \text { if } k=2 n-4 ; \\ \sum_{j=0}^{k} r_{i} r_{k-i}+(3 n-6-k+1), & \text { if } 2 n-4<k \leq 3 n-6 ; \\ \sum_{i=0}^{k} r_{i} r_{k-i}, & \text { if } 0 \leq k<n-2 .\end{cases}
$$

To show the injectivity of the cycle class map $\mathrm{R}^{*}(F \times F) \rightarrow \mathrm{H}^{2 *}(F \times F)$, we bound from below the dimension of the image in each degree by computing the dimension of Hodge classes on $F \times F$ for $X$ very general.

Lemma 5.12 Let $F$ be the Fano variety of lines on a very general cubic hypersurface $X \subseteq \mathbb{P}^{n+1}$. Then

$$
\begin{aligned}
& \operatorname{hdg}\left(\mathrm{H}^{2 i}(F)\right)=r_{i}, \text { for any } i \text { and } \\
& \operatorname{hdg}\left(\mathrm{H}^{2 k}(F \times F)\right)= \begin{cases}\sum_{i=0}^{k} r_{i} r_{k-i}, & \text { if } 0 \leq k<n-2 ; \\
\sum_{i=0}^{k} r_{i} r_{k-i}+(k-(n-2)+1), & \text { if } n-2 \leq k<2 n-4 ; \\
\sum_{i=0}^{2 n-4} r_{i} r_{k-i}+(n-1)+1, & \text { if } k=2 n-4 ; \\
\sum_{i=0}^{k} r_{i} r_{k-i}+(3 n-6-k+1), & \text { if } 2 n-4<k \leq 3 n-6 ; \\
\sum_{i=0}^{k} r_{i} r_{k-i}, & \text { if } 0 \leq k<n-2 .\end{cases}
\end{aligned}
$$

Moreover, if $n \neq 2$, the cycle class map induces surjective morphisms

$$
\operatorname{GDCH}^{*}(F) \longrightarrow \operatorname{Hdg}\left(\mathrm{H}^{*}(F)\right) \text { and } \operatorname{GDCH}^{*}(F \times F) \longrightarrow \operatorname{Hdg}\left(\mathrm{H}^{*}(F \times F)\right) .
$$

In particular, (18) is an equality.

Proof The Hodge structure $\mathrm{H}^{*}(F, \mathbb{Q})$ was computed, for the Fano variety of lines on any smooth cubic hypersurface, in terms of $H_{X}:=\mathrm{H}_{\text {prim }}^{n}(X)(1)$ by Galkin-Shinder [22, Theorem 6.1]: 


$$
\mathrm{H}^{*}(F) \simeq \operatorname{Sym}^{2} H_{X} \oplus \bigoplus_{i=0}^{n-2} H_{X}(-i) \oplus \bigoplus_{i=0}^{2 n-4} \mathbb{Q}(-i)^{a_{i}},
$$

where $a_{i}=r_{i}$ except for $a_{n-2}=r_{n-2}-1$. (This can be seen by applying $\mathrm{H}^{*}$ to the isomorphism (16) and using the semi-simplicity of the category of polarizable Hodge structures). The computation of $\operatorname{hdg}\left(\mathrm{H}^{2 *}(F)\right)$ and $\operatorname{hdg}\left(\mathrm{H}^{2 *}(F \times F)\right)$ is then straightforward from Lemma 5.5.

We now show that for $X$ very general of dimension $\neq 2$, the cycle class map $\mathrm{GDCH}^{*}(F) \rightarrow \operatorname{Hdg}\left(\mathrm{H}^{*}(F)\right)$ is surjective. The proof is similar to that of Proposition 5.11. With the notation therein, we know that the cokernels of $\mathrm{GDCH}^{*}(M) \rightarrow \mathrm{H}^{*}(M)$ and $\mathrm{GDCH}^{*}(N) \rightarrow \mathrm{H}^{*}(N)$ are trivial by Proposition 5.6. It follows for dimension reasons that the cokernel of $\operatorname{GDCH}^{*}\left(P_{2}\right) \rightarrow \mathrm{H}^{*}\left(P_{2}\right)$ is trivial, i.e., that the cycle class map $\mathrm{GDCH}^{*}(F) \rightarrow \operatorname{Hdg}\left(\mathrm{H}^{*}(F)\right)$ is surjective.

Finally, by Lemma 5.5, to see that the cycle class map $\operatorname{GDCH}^{*}(F \times F) \rightarrow \operatorname{Hdg}\left(\mathrm{H}^{*}(F \times F)\right)$ is surjective for $X$ very general of dimension $\neq 2$, it suffices to observe that the extra class appearing in $\mathrm{Sym}^{2} H_{X} \otimes \mathrm{Sym}^{2} H_{X}$ is accounted for by the diagonal $\Delta_{F}$.

Now we can conclude the proof of Theorem 5.2. The surjectivity of the restriction of the cycle class maps to generically defined cycles on $F$ or $F \times F$ was treated in Lemma 5.12. We note that the Franchetta property for $F$ and $F \times F$ in case $\operatorname{dim} X \leq 2$ is trivial; since then, $F$ is either empty or zero-dimensional. Assume now that $\operatorname{dim} X>2$. For a very general cubic hypersurface $X$ and any integer $k$, consider the cycle class map

$$
\mathrm{R}^{k}(F \times F) \rightarrow \mathrm{H}^{2 k}(F \times F) .
$$

On the one hand, by (17), $\mathrm{R}^{k}(F \times F)$ consists of the generically defined cycles $\mathrm{GDCH}^{k}(F \times F)$, and hence, the cycle class map is onto the space of algebraic classes in $\mathrm{H}^{2 k}(F \times F)$; on the other hand, by comparing (21) and Lemma 5.12, we see that the upper bound for the dimension of $\mathrm{R}^{k}(F \times F)$ coincides with the dimension of space of algebraic classes in $\mathrm{H}^{2 k}(F \times F)$. Therefore, (21) are equalities and the cycle class map is injective. A specialization argument then shows that the Franchetta property holds for $\mathcal{F} \times{ }_{B} \mathcal{F} \rightarrow B$, thereby concluding the proof of Theorem 5.2.

\subsection{Proof of Theorem 5.4}

Let $\pi_{\mathcal{X}}^{i} \in \mathrm{CH}^{n}\left(\mathcal{X} \times_{B} \mathcal{X}\right)$ be the relative Chow-Künneth projectors of (4). We have to show that the cycle

$$
\pi_{\mathcal{X}}^{k} \circ \delta_{\mathcal{X} / B} \circ\left(\pi_{\mathcal{X}}^{i} \otimes \pi_{\mathcal{X}}^{j}\right) \in \mathrm{CH}^{2 n}\left(\mathcal{X} \times_{B} \mathcal{X} \times_{B} \mathcal{X}\right)
$$

vanishes fiberwise for all $k \neq i+j$. Let $\sigma$ be the relative morphism $\mathcal{X} \times_{B} \mathcal{X} \rightarrow \mathcal{X} \times_{B} \mathcal{X}$ that permutes the factors. Then, by commutativity of the algebra structure on the motive of a variety, we have

$$
\pi_{\mathcal{X}}^{k} \circ \delta_{\mathcal{X} / B} \circ\left(\pi_{\mathcal{X}}^{i} \otimes \pi_{\mathcal{X}}^{j}\right) \circ \sigma=\pi_{\mathcal{X}}^{k} \circ \delta_{\mathcal{X} / B} \circ \sigma \circ\left(\pi_{\mathcal{X}}^{j} \otimes \pi_{\mathcal{X}}^{i}\right)=\pi_{\mathcal{X}}^{k} \circ \delta_{\mathcal{X} / B} \circ\left(\pi_{\mathcal{X}}^{j} \otimes \pi_{\mathcal{X}}^{i}\right)
$$

as cycles in $\mathrm{CH}^{2 n}\left(\mathcal{X} \times_{B} \mathcal{X} \times_{B} \mathcal{X}\right)$. It follows that the pull-push of (23) along $\mathcal{X} \times_{B} \mathcal{X} \times_{B} \mathcal{X} \rightarrow \mathcal{X}^{(2)} \times_{B} \mathcal{X}$ is the cycle 


$$
\pi_{\mathcal{X}}^{k} \circ \delta_{\mathcal{X} / B} \circ\left(\pi_{\mathcal{X}}^{i} \otimes \pi_{\mathcal{X}}^{j}+\pi_{\mathcal{X}}^{j} \otimes \pi_{\mathcal{X}}^{i}\right) \in \mathrm{CH}^{2 n}\left(\mathcal{X} \times_{B} \mathcal{X} \times_{B} \mathcal{X}\right)
$$

Since the cycles (23) vanish cohomologically fiberwise for all $k \neq i+j$, the same holds for the cycles (24) for all $k \neq i+j$. Combining Proposition 5.10 with Theorem 5.2, we find that the cycles (24) vanish in the Chow group fiberwise for all $k \neq i+j$. Composing on the right with $\pi_{\mathcal{X}}^{i} \otimes \pi_{\mathcal{X}}^{j}$ proves the theorem.

Remark 5.13 (Proof of Theorem 5.4 using [13, Corollary 3.3.9]) Let $X$ be a fiber of $\mathcal{X} \rightarrow B$. Using (8), one can see that the cycle $\pi_{X}^{k} \circ \delta_{X} \circ\left(\pi_{X}^{i} \otimes \pi_{X}^{j}\right) \in \mathrm{CH}^{2 n}(X \times X \times X)$ belongs to the subring $\mathrm{R}^{*}(X \times X \times X):=\left\langle p_{i}^{*} h, p_{j k}^{*} \Delta_{X}\right\rangle$, where $h$ is the restriction of $H$ to the fiber $X$ and where $p_{i}$ and $p_{j k}$ are the natural projections. It follows that the cycle $\pi_{X}^{k} \circ \delta_{X} \circ\left(\pi_{X}^{i} \otimes \pi_{X}^{j}\right)+\pi_{X}^{k} \circ \delta_{X} \circ\left(\pi_{X}^{j} \otimes \pi_{X}^{i}\right)$ belongs to $\mathrm{R}^{*}\left(X^{(2)} \times X\right):=\mathrm{R}^{*}(X \times X \times X)^{\mathfrak{S}_{2}}$, where $\mathfrak{S}_{2}$ is the symmetric group acting by permuting the first two factors. Diaz shows in [13, Corollary 3.3.9] that the latter subring injects in cohomology. This yields that the cycle (24) vanishes fiberwise for all $k \neq i+j$ and one concludes as before, by composing on the right with $\pi_{\mathcal{X}}^{i} \otimes \pi_{\mathcal{X}}^{j}$, that the cycle (23) vanishes fiberwise for all $k \neq i+j$, thereby establishing Theorem 5.4.

Remark 5.14 (Chern classes) By definition of $\pi_{\mathcal{X}}^{2}$, we have for a smooth cubic fourfold $X$ that $\mathrm{CH}^{1}(X)_{(0)}=\mathrm{CH}^{1}(X)=\mathbb{Q} h$. Since the Chern classes of $X$ are powers of $h$, we get by multiplicativity that

$$
c_{i}(X) \in \mathrm{CH}^{i}(X)_{(0)}
$$

for all $i$. This is especially useful in view of Proposition 2.9.

Remark 5.15 (Uniqueness of the MCK decomposition) Let $X$ be a smooth cubic fourfold whose Chow motive is finite-dimensional, in the sense of Kimura [29]. Then $X$ admits a unique MCK decomposition. Indeed, let $\left\{\pi_{X}^{i}, 0 \leq i \leq 8\right\}$ be a Chow-Künneth decomposition for $X$. By Kimura finite-dimensionality, since $X$ has vanishing odd cohomology, we must have $\pi_{X}^{i}=0$ for all $i$ odd. Since $\mathrm{CH}_{0}(X)=\mathbb{Q} h^{4}$, we have by Remark 2.2 that $\pi_{X}^{0}=\frac{1}{3} h^{4} \times 1_{X}$. Since $\mathrm{H}^{2}(X)=\mathbb{Q}(-1)=\mathbb{Q} h$, we have $\mathfrak{h}^{2}(X) \simeq \mathbb{1}(-1)$. In other words, $\pi_{X}^{2}$ factors as $\mathfrak{h}(X) \rightarrow \mathbb{1}(-1) \rightarrow \mathfrak{h}(X)$. Since $\mathrm{CH}^{1}(X)=\mathbb{Q} h$, we see that necessarily $\pi_{X}^{2}=\frac{1}{3}\left(h^{3}+\varepsilon\right) \times h$, where $\varepsilon$ is a homologically trivial 1-cycle on $X$. Suppose now that $\left\{\pi_{X}^{i}, 0 \leq i \leq 8\right\}$ is an MCK decomposition. Then by Proposition 2.7 we know it must be self-dual. In particular, we get $\pi_{X}^{8}=\frac{1}{3} 1_{X} \times h^{4}$ and $\pi_{X}^{6}=\frac{1}{3} h \times\left(h^{3}+\varepsilon\right)$. By multiplicativity, we must have $\mathrm{CH}^{3}(X)_{(0)}=\left(\mathrm{CH}^{1}(X)_{(0)}\right)^{\cdot 3}=\mathbb{Q} h^{3}$; this implies that $\varepsilon=0$, and therefore establishes the uniqueness claim.

Let now $X$ be a cubic threefold. It is known that $\mathrm{CH}_{0}(X)=\mathbb{Q} h^{3}$ and consequently that $\mathfrak{h}(X)$ is finite-dimensional in the sense of Kimura. The above arguments then establish unconditionally that $X$ admits a unique MCK decomposition. Likewise, if $X$ is a cubic fivefold, then it is known that $\mathrm{CH}_{0}(X)=\mathbb{Q} h^{5}$ and $\mathrm{CH}_{1}(X)=\mathbb{Q} h^{4}$ (see [54]) and consequently [67, Example 4.12] that $\mathfrak{h}(X)$ is finite-dimensional in the sense of Kimura. The reader will have no trouble adapting the above arguments to show unconditionally that cubic threefolds and cubic fivefolds admit a unique MCK decomposition. 


\section{Küchle fourfolds of type $c 7$}

As a first step towards the construction of Fano fourfolds, Küchle classified in [30] all Fano fourfolds of index 1 that can be obtained as zero loci of generic sections of homogenous vector bundles on Grassmannian varieties. Among those Küchle fourfolds, those with type $c 5, c 7, d 3$ are of cohomological $\mathrm{K} 3$ type. The aim of this section is to establish the existence of an MCK decomposition for Küchle fourfolds of type $c 7$. Those of type $d 3$ were dealt with in [38], while the case of those of type $c 5$ is still open and certainly worth further study; see [33]. Let us first recall the definition.

Definition 6.1 [30, Theorem 3.1] A Küchle fourfold of type $c 7$ is the zero locus of a generic section of the vector bundle

$$
\wedge^{2} \mathcal{Q}(1) \oplus \mathcal{O}(1)
$$

on the Grassmannian $\operatorname{Gr}(3,8)$, where $\mathcal{Q}$ is the tautological rank-5 quotient bundle and $\mathcal{O}(1):=\operatorname{det} \mathcal{Q}$ is the Plücker line bundle.

Küchle fourfolds of type $c 7$ are of cohomological K3 type; their Hodge diamonds are as follows:

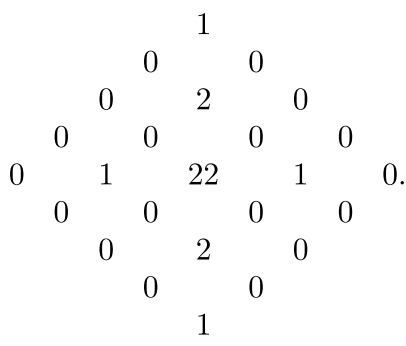

The main result of this section is the following.

Theorem 6.2 A Küchle fourfold X of type c7 has an MCK decomposition. Moreover, the Chern classes $c_{j}(X)$ are in $\mathrm{CH}^{*}(X)_{(0)}$.

We need an alternative description, due to Kuznetsov [32, Section 4], of Küchle fourfolds of type $c 7$ as blow-ups of (special) cubic fourfolds along the Veronese surface. Let $M$ be the blow-up of $\mathbb{P}^{5}$ along the Veronese surface $S:=v_{2}\left(\mathbb{P}^{2}\right)$, where $v_{2}: \mathbb{P}^{2} \rightarrow \mathbb{P}^{5}$ is the embedding induced by the linear system $\left|\mathcal{O}_{\mathbb{P}^{2}}(2)\right|^{2}$ Let $\pi: M \rightarrow \mathbb{P}^{5}$ be the blow-up morphism. It was shown in [32, Corollary 4.11] that a generic Küchle fourfold $X$ of type $c 7$ arises as a member of the linear system $|3 H-E|$ on $M$, where $H$ is the pullback of the hyperplane section class on $\mathbb{P}^{5}$ and $E$ is the exceptional divisor. Therefore, its image $Y:=\pi(X)$ is a cubic fourfold containing $S$. Set $\bar{B}:=\mathbb{P H}^{0}\left(M, \mathcal{O}_{M}(3 H-E)\right)$, and set $B$ to be the Zariski open subset of $\bar{B}$ parameterizing smooth fourfolds $X_{b} \subset M$ such that the

\footnotetext{
${ }^{2}$ In [32, Section 4], $M$ was defined to be the zero locus of a generic section of the vector bundle $\wedge^{3} \mathcal{Q}$ on $\operatorname{Gr}(3,8)$. But according to [32, Theorem 4.10], this is equivalent to the definition by blow-up.
} 
cubic fourfold $Y_{b}=\pi\left(X_{b}\right)$ is also smooth. Let $\mathcal{X} \subset M \times B$ and $\mathcal{Y} \subset \mathbb{P}^{5} \times B$ denote the universal families of Küchle fourfolds of type $c 7$ and cubic fourfolds, respectively.

As a first step of the proof, we establish the Franchetta property for these two families, which is of independent interest. Note that $\mathcal{Y} \rightarrow B$ is the universal family of Hassett's special cubic fourfolds $\mathcal{C}_{20}$.

Lemma 6.3 The families $\mathcal{X} \rightarrow B$ and $\mathcal{Y} \rightarrow B$ have the Franchetta property, in the sense of Definition 5.1.

Proof Let $\overline{\mathcal{X}} \rightarrow \bar{B}$ denote the family of possibly singular hypersurfaces. Since the linear system $|3 H-E|$ is base point-free, $p: \overline{\mathcal{X}} \rightarrow M$ has the structure of a projective bundle. We first show the following equality:

$$
\operatorname{Im}\left(\mathrm{CH}^{*}(\overline{\mathcal{X}}) \rightarrow \mathrm{CH}^{*}\left(X_{b}\right)\right)=\operatorname{Im}\left(\mathrm{CH}^{*}(M) \rightarrow \mathrm{CH}^{*}\left(X_{b}\right)\right), \quad \forall b \in B .
$$

Indeed, for any $\alpha \in \mathrm{CH}^{i}(\overline{\mathcal{X}})$, the projective bundle formula yields

$$
\alpha=\sum_{j=0}^{i} p^{*}\left(a_{i-j}\right) \cdot \xi^{j} \quad \text { in } \mathrm{CH}^{i}(\overline{\mathcal{X}}),
$$

where $\xi \in \mathrm{CH}^{1}(\overline{\mathcal{X}})$ is relatively ample with respect to $p$ and $a_{j} \in \mathrm{CH}^{j}(M)$. Let $h \in \mathrm{CH}^{1}(\bar{B})$ be a hyperplane section, and let $q: \overline{\mathcal{X}} \rightarrow \bar{B}$ denote the projection. We have $q^{*}(h)=v \xi+p^{*}(z)$, for some $v \in \mathbb{Q}$ and $z \in \mathrm{CH}^{1}(M)$. It is readily checked that $v$ is nonzero. (Indeed, assume for a moment $v$ were zero. Then we would have $q^{*}\left(h^{\operatorname{dim} \bar{B}}\right)=p^{*}\left(z^{\operatorname{dim} \bar{B}}\right)$ in $\mathrm{CH}^{\operatorname{dim} \bar{B}}(\overline{\mathcal{X}})$. But the right-hand side is zero, since $\operatorname{dim} \bar{B}>\operatorname{dim} M=5$, while the left-hand side is nonzero.)

The constant $v$ being nonzero, we can write

$$
\xi=p^{*}(z)+q^{*}(c) \quad \text { in } \mathrm{CH}^{1}(\overline{\mathcal{X}}),
$$

where $z \in \mathrm{CH}^{1}(M)$ and $c \in \mathrm{CH}^{1}(\bar{B})$ are nonzero elements. The restriction of $q^{*}(c)$ to a fiber $X_{b}$ is zero, and so, we find that

$$
\left.\alpha\right|_{X_{b}}=\left.a_{i}^{\prime}\right|_{X_{b}} \text { in } \mathrm{CH}^{i}\left(X_{b}\right)
$$

for some $a_{i}^{\prime} \in \mathrm{CH}^{i}(M)$. This proves the equality (25).

Second, we claim that for any $b \in B$, we have the following equality:

$$
\operatorname{Im}\left(\mathrm{CH}^{*}\left(\mathcal{Y} \rightarrow Y_{b}\right)\right)=\operatorname{Im}\left(\mathrm{CH}^{*}\left(\mathbb{P}^{5}\right) \rightarrow \mathrm{CH}^{*}\left(Y_{b}\right)\right)+\operatorname{Im}\left(\tau_{b, *}: \mathrm{CH}^{*}(S) \rightarrow \mathrm{CH}^{*}\left(Y_{b}\right)\right),
$$

where $\tau_{b}: S \rightarrow Y_{b}$ is the natural inclusion and the other morphisms are natural restriction maps. The right-hand side of (26) is clearly contained in its left-hand side. Let us show the reverse inclusion. Denote by $\pi_{b}: X_{b} \rightarrow Y_{b}$ the restriction of $\pi$ to $X_{b}$, which fits into the following Cartesian commutative diagram: 


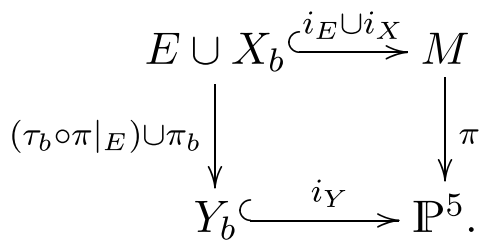

For any $z \in \operatorname{Im}\left(\mathrm{CH}^{*}\left(\mathcal{Y} \rightarrow Y_{b}\right)\right), z=\pi_{b, *}\left(\pi_{b}^{*}(z)\right)=\pi_{b, *}\left(i_{X}^{*}(\alpha)\right)$ for some $\alpha \in \mathrm{CH}^{*}(M)$. Here, we used (25) and the fact that $\pi_{b}^{*}(z) \in \mathrm{CH}^{*}\left(X_{b}\right)$ is the restriction of a cycle of $\mathcal{X}$. By the base change formula, we obtain

$$
i_{Y}^{*} \pi_{*}(\alpha)=\pi_{b, *}\left(i_{X}^{*}(\alpha)\right)+\tau_{b, *}\left(\left.\pi\right|_{E}\right)_{*}\left(i_{E}^{*}(\alpha)\right),
$$

where the first term on the right-hand side is $z$. The equality (26) is proved.

Denote as before $\operatorname{GDCH}^{*}\left(Y_{b}\right):=\operatorname{Im}\left(\mathrm{CH}^{*}\left(\mathcal{Y} \rightarrow Y_{b}\right)\right)$, the "generically defined cycles." Thanks to (26), $\operatorname{GDCH}^{*}\left(Y_{b}\right)$ is generated, as a $\mathbb{Q}$-subalgebra of $\mathrm{CH}^{*}\left(Y_{b}\right)$, by the hyperplane class $h$, the fundamental class of the Veronese surface $S$ and the class of a line $l$ in the surface $S$. We need to show that the cycle class map restricted to this subalgebra $\operatorname{GDCH}^{*}\left(Y_{b}\right)=\langle h, S, l\rangle$ is injective.

To this end, we first observe that the generator $l$ is redundant:

$$
l:=\tau_{*}\left(c_{1}\left(\mathcal{O}_{S}(1)\right)\right)=\tau_{*}\left(\frac{1}{2} \tau^{*}(h)\right)=\frac{1}{2} h \cdot S .
$$

Hence, $\operatorname{GDCH}^{*}\left(Y_{b}\right)=\langle h, S\rangle$. The cohomology classes $[S]$ and $[h]^{2}$ being independent, we only need to establish the following two relations in $\mathrm{CH}^{*}\left(Y_{b}\right)$ :

- $S^{2}$ is proportional to $h^{4}$;

- $S \cdot h$ is proportional to $h^{3}$.

The first one follows immediately from the fact that $\mathrm{CH}_{0}\left(Y_{b}\right) \simeq \mathbb{Q}$. For the second one, using the fact that the class of the Veronese surface in $\mathbb{P}^{5}$ is $4 c_{1}\left(\mathcal{O}_{\mathbb{P}^{5}}(1)\right)^{3}$, we have

$$
S \cdot h=\frac{1}{3} i_{Y}^{*}\left(i_{Y, *}(S)\right)=\frac{4}{3} h^{3},
$$

where $i_{Y}: Y_{b} \rightarrow \mathbb{P}^{5}$ is the natural inclusion.

The Franchetta property for $\mathcal{X} \rightarrow B$ now follows from the commutative diagram

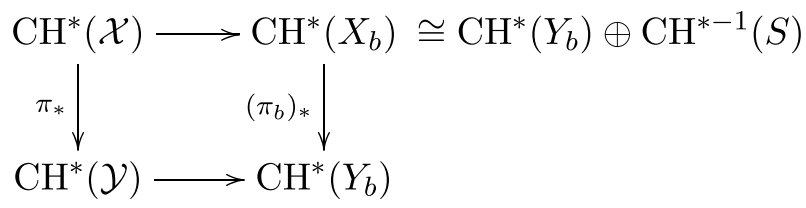

plus the fact that the Veronese surface $S \cong \mathbb{P}^{2} \subset Y_{b}$ has trivial Chow groups.

Proof (Proof of Theorem 6.2) To prove that Küchle fourfolds of type $c 7$ have an MCK decomposition, it suffices, by specialization, to show that the generic Küchle fourfold of 
type $c 7$ has an MCK decomposition with universally defined projectors. The generic fourfold of type $c 7$ is a blow-up of a cubic fourfold as above, and so to establish an MCK, it suffices to check that the hypotheses of the "blow-up" part of Proposition 2.9 are met with. The second, third and fourth hypotheses follow from Theorem 5.4, Remark 5.14, and the fact that $S \cong \mathbb{P}^{2}$. The only thing that needs checking is the first hypothesis, i.e., that the graph of the inclusion morphism $\tau: S \rightarrow Y$ lies in $\mathrm{CH}^{*}(S \times Y)_{(0)}$.

Clearly, the inclusion morphism is universally defined (i.e., it exists as a relative morphism $\tau$ over the base $B$ ). Since

$$
\Gamma_{\tau_{b}} \in \mathrm{CH}^{4}\left(S \times Y_{b}\right) \cong \bigoplus_{j=2}^{4} \mathrm{CH}^{j}\left(Y_{b}\right)
$$

and since the MCK decomposition for $S \times Y_{b}$ is universally defined over the base $B$, it follows from Lemma 6.3 that

$$
\left(\pi_{S \times Y_{b}}^{j}\right)_{*}\left(\Gamma_{\tau_{b}}\right)=0 \quad \text { in } \mathrm{CH}^{4}\left(S \times Y_{b}\right) \quad \forall j \neq 8,
$$

i.e., $\Gamma_{\tau_{b}} \in \mathrm{CH}^{4}\left(S \times Y_{b}\right)_{(0)}$, as desired.

Finally, the statement about the Chern classes $c_{j}(X)$ follows from Lemma 6.3, in view of the fact that the Chern classes of $X$ and the MCK decomposition of $X$ are universally defined.

Remark 6.4 The link between Küchle $c 7$ and cubic fourfolds is interesting also on the cubic fourfolds side. Indeed, thanks to the relation with Küchle $c 7$ we were able to show (Lemma 6.3) the Franchetta property for the Hassett divisor $\mathcal{C}_{20}$ (parameterizing cubic fourfolds containing a Veronese surface). More generally, one can ask whether all Hassett divisors $\mathcal{C}_{d}$ have the Franchetta property. For those values of $d$ where there is an associated K3 surface, this is equivalent to O'Grady's generalized Franchetta conjecture for K3 surfaces. For other values of $d$ (such as $d=20$ ), the expected answer is not so clear.

\section{Todorov surfaces}

We exhibit examples of regular surfaces with ample canonical class and with cohomology of K3 type that admit a multiplicative Chow-Künneth decomposition, namely Todorov surfaces of type $(0,9)$ and $(1,10)$. For that purpose, we first state a general criterion for lifting MCK decompositions along dominant morphisms of regular surfaces. (Criteria for descending an MCK decomposition along a generically finite morphism were given and used in $[60,61]$ to show that MCK decompositions were stable under the operation of taking the Hilbert scheme of length-2 or 3 subschemes.) We then define Todorov surfaces and check that for those of type $(0,9)$ and $(1,10)$ all the hypotheses of the general criterion are met with. As a by-product, we obtain a Franchetta-type result for those Todorov surfaces.

\subsection{A general criterion for lifting MCK decompositions on regular surfaces}

Let $p: S \rightarrow T$ be a dominant morphism between regular surfaces. In Remark 4.3, we saw that an MCK decomposition on $S$ descends to give an MCK decomposition on $T$. In general, one cannot infer an MCK decomposition on $S$ from an MCK decomposition on $T$. 
(Consider indeed the blow-up of a K3 surface at a very general point.) The following proposition provides sufficient conditions for an MCK decomposition on $T$ to lift to an MCK decomposition on $S$ and will be used to construct such a decomposition on certain Todorov surfaces.

Proposition 7.1 (Lifting MCK decompositions) Let $\mathcal{T} \rightarrow B$ and $\mathcal{S} \rightarrow B$ be relatively flat and projective surfaces over a smooth quasi-projective complex variety $B$. For any $b \in B$, denote by $T_{b}$ and $S_{b}$ the respective fibers. Assume that for all $b \in B, S_{b}$ and $T_{b}$ are regular surfaces which are smooth or finite group quotients of smooth surfaces. Let $p: \mathcal{S} \rightarrow \mathcal{T}$ be a dominant morphism over B. Assume the following conditions:

(i) (Franchetta property for $\mathcal{T} / B)$ There exists a cycle $c \in \mathrm{CH}^{2}(\mathcal{T})$ which is fiberwise of degree 1 such that for any $\alpha \in \mathrm{CH}^{2}(\mathcal{T})$ and for any $b \in B$, we have $\alpha_{b} \in \mathbb{Q} c_{b}$;

(ii) The relative $C K$ decomposition $\pi_{\mathcal{T}}^{0}=c \times_{B} \mathcal{T}, \pi_{\mathcal{T}}^{4}=\mathcal{T} \times_{B} c, \pi_{\mathcal{T}}^{2}=\Delta_{\mathcal{T} / B}-\pi_{\mathcal{T}}^{0}-\pi_{\mathcal{T}}^{4}$ is fiberwise an MCK decomposition; in other words, the modified small diagonal vanishes $\Gamma_{3}\left(T_{b}, c_{b}\right)=0$ for any $b \in B$ (see Proposition 4.1$)$.

(iii) For any $b \in B$, the pushforward $p_{*}: \mathrm{CH}^{2}\left(S_{b}\right) \rightarrow \mathrm{CH}^{2}\left(T_{b}\right)$ is an isomorphism.

Then, denoting $c^{\prime}=\frac{1}{\operatorname{deg}(p)} p^{*} c$, the set $\left\{\pi_{\mathcal{S}}^{0}=c^{\prime} \times_{B} \mathcal{S}, \pi_{\mathcal{S}}^{4}=\mathcal{S} \times_{B} c^{\prime}, \pi_{\mathcal{S}}^{2}=\Delta_{\mathcal{S} / B}-\pi_{\mathcal{S}}^{0}-\pi_{\mathcal{S}}^{4}\right\}$ defines a relative $C K$ decomposition for $\mathcal{S}$ which is fiberwise an MCK decomposition. Moreover, the Franchetta property holds for the family $\mathcal{S} \rightarrow B$.

Proof Intersection theory with rational coefficients extends naturally to quotients of smooth varieties by finite groups. Denote by $d:=\operatorname{deg}(p)$. First of all, note that the inverse of the isomorphism in (iii) is obviously $\frac{1}{d} p^{*}$ by the projection formula. Then we can establish the Franchetta property for the family $\mathcal{S} / B$ : for any $\alpha \in \mathrm{CH}^{2}(\mathcal{S})$ and any $b \in B$, by $(i)$, $p_{*}\left(\alpha_{b}\right)=m c_{b}$ in $\mathrm{CH}_{0}\left(T_{b}\right)$ for some $m \in \mathbb{Q}$. Therefore,

$$
\alpha_{b}=\frac{1}{d} p^{*}\left(p_{*}\left(\alpha_{b}\right)\right)=\frac{1}{d} p^{*}\left(m c_{b}\right)=m c_{b}^{\prime} \in \mathbb{Q} c_{b}^{\prime} .
$$

To show the multiplicativity of the Chow-Künneth decomposition for the fibers of $\mathcal{S}$, we need to reinterpret it as follows. The relative Chow motive of $\mathcal{S} / B$ splits as

$$
\mathfrak{h}(\mathcal{S})=\mathfrak{h}(\mathcal{S})^{\text {inv }} \oplus \mathfrak{h}(\mathcal{S})^{\text {coinv }},
$$

where $\mathfrak{h}(\mathcal{S})^{\text {inv }}=\frac{1}{d} p^{*} p_{*} \mathfrak{h}(\mathcal{S})$ and $\mathfrak{h}(\mathcal{S})^{\text {coinv }}=\left(\Delta_{\mathcal{S} / B}-\frac{1}{d} p^{*} p_{*}\right) \mathfrak{h}(\mathcal{S})$. For any $b \in B, \mathfrak{h}\left(S_{b}\right)^{\text {inv }}$ forms a subalgebra object of $\mathfrak{h}\left(S_{b}\right)$, because $\frac{1}{d} p^{*} p_{*} \circ \delta_{S_{b}} \circ\left(\frac{1}{d} p^{*} p_{*} \otimes \frac{1}{d} p^{*} p_{*}\right)=$ $\delta_{S_{b}} \circ\left(\frac{1}{d} p^{*} p_{*} \otimes \frac{1}{d} p^{*} p_{*}\right)$. Moreover, $p^{*}$ induces an isomorphism of algebra objects:

$$
\mathfrak{h}\left(T_{b}\right) \simeq \mathfrak{h}\left(S_{b}\right)^{\text {inv }} .
$$

In particular, $p_{*}: \mathrm{CH}^{2}\left(\mathfrak{h}\left(S_{b}\right)^{\text {inv }}\right) \rightarrow \mathrm{CH}^{2}\left(\mathfrak{h}\left(T_{b}\right)\right)$ is an isomorphism. The assumption (iii) implies that for any $b \in B, \mathrm{CH}^{2}\left(\mathfrak{h}\left(S_{b}\right)^{\text {coinv }}\right)=0$. It is also clear that $\mathrm{CH}^{0}\left(\mathfrak{h}\left(S_{b}\right)^{\text {coinv }}\right)=0$. By Lemma 7.2, $\mathfrak{h}\left(S_{b}\right)^{\text {coinv }}$ is isomorphic to $\mathbb{1}(-1)^{r}$ for some $r \in \mathbb{N}$. Note that the number $r$ may vary with $b$, but the minimal value is attained by a very general point $b$.

Now it is easy to see that fiberwise the relative Chow-Künneth decomposition for $\mathcal{S} / B$ is given as $\mathfrak{h}^{i}\left(S_{b}\right)=\mathfrak{h}^{i}\left(T_{b}\right)$ for $i=0$ and 4, via the isomorphism (28), while $\mathfrak{h}^{2}\left(S_{b}\right)=\mathfrak{h}^{2}\left(T_{b}\right) \oplus \mathfrak{h}\left(S_{b}\right)^{\text {coinv }}$. Let us show that it is multiplicative. Let $b$ be a very general point of $B$. 
- By the isomorphism (28) and the assumption (ii), this decomposition is multiplicative on the subalgebra summand $\mathfrak{h}\left(S_{b}\right)^{\text {inv }}$.

- By construction, the intersection product sends $\mathfrak{h}\left(S_{b}\right)^{\text {coinv }} \otimes \mathfrak{h}\left(S_{b}\right)^{\text {coinv }}$ to $\mathfrak{h}\left(S_{b}\right)^{\text {inv }}$ and we need to show that this map lands in $\mathfrak{h}^{4}\left(S_{b}\right)$. As $\mathfrak{h}\left(S_{b}\right)^{\text {coinv }} \simeq \mathbb{1}(-1)^{r}$, the morphism $\mathfrak{h}\left(S_{b}\right)^{\text {coinv }} \otimes \mathfrak{h}\left(S_{b}\right)^{\text {coinv }} \rightarrow \mathfrak{h}\left(S_{b}\right)^{\text {inv }}$ is given by $r^{2}$ elements of $\mathrm{CH}^{2}\left(\mathfrak{h}\left(S_{b}\right)^{\text {inv }}\right)$. Clearly each of these 0 -cycles is the restriction to the fiber of an element of $\mathrm{CH}^{2}(\mathcal{S})$. By the Franchetta property established in the beginning of the proof, this element is fiberwise a multiple of $c_{b}^{\prime}$. We conclude by the definition of $\mathfrak{h}^{4}\left(S_{b}\right)$.

- Similarly, the intersection product sends $\mathfrak{h}\left(S_{b}\right)^{\text {inv }} \otimes \mathfrak{h}\left(S_{b}\right)^{\text {coinv }}$ to $\mathfrak{h}\left(S_{b}\right)^{\text {coinv }}$. From the fact that $\mathfrak{h}\left(S_{b}\right)^{\text {coinv }}$ is a direct sum of Lefschetz motives, one sees immediately that the intersection product sends $\mathfrak{h}^{0}\left(S_{b}\right) \otimes \mathfrak{h}\left(S_{b}\right)^{\text {coinv }}$ to $\mathfrak{h}\left(S_{b}\right)^{\text {coinv }}$ (the fundamental class is a unit for the algebra structure on the motive of a variety) and sends $\mathfrak{h}^{4}\left(S_{b}\right) \otimes \mathfrak{h}\left(S_{b}\right)^{\text {coinv }}$ to zero. It remains to show that the intersection product sends $\mathfrak{h}^{2}\left(S_{b}\right)^{\text {inv }} \otimes \mathfrak{h}\left(S_{b}\right)^{\text {coinv }}$ to zero. To this end, we use again that $\mathfrak{h}\left(S_{b}\right)^{\text {coinv }} \simeq \mathbb{1}(-1)^{r}$ to see that the morphism $\mathfrak{h}^{2}\left(S_{b}\right)^{\text {inv }} \otimes \mathfrak{h}\left(S_{b}\right)^{\text {coinv }} \rightarrow \mathfrak{h}\left(S_{b}\right)^{\text {coinv }}$ is given by $r^{2}$ elements of $\operatorname{Hom}\left(\mathfrak{h}^{2}\left(S_{b}\right)^{\text {inv }}, \mathbb{1}\right)$, which is a subspace of $\mathrm{CH}^{2}\left(S_{b}\right)_{\mathrm{deg}=0}$. Similarly as in the previous item, each of these 0 -cycles is the restriction to the fiber of an element of $\mathrm{CH}^{2}(\mathcal{S})$ and the Franchetta property for $\mathcal{S} / B$ tells us that these elements are zero.

The following easy lemma, which is a special case of [69, Cor. 2.2] in the case $\pi={ }^{t} \pi$, is used in the proof of the previous proposition. We provide a proof for the sake of completeness.

Lemma 7.2 Let $S$ be a smooth projective regular surface and $\pi \in \mathrm{CH}^{2}(S \times S)$ be a projector. If the Chow motive $M:=(S, \pi)$ has vanishing $\mathrm{CH}^{2}$ and $\mathrm{CH}^{0}$, then $M$ is isomorphic to a direct sum of copies of the Lefschetz motive $1(-1)$.

Proof The condition $\mathrm{CH}^{2}(M)=0$ implies that for any $x \in S$, the restriction of $\pi$ to the fiber $\{x\} \times S$ is zero in $\mathrm{CH}^{2}(S)$. By the Bloch-Srinivas decomposition of diagonal theorem [7], there exists a divisor $D$ of $S$, such that $\pi$ is supported in $D \times S$. As $S$ is assumed to be regular, $\pi$ must be of the form

$$
\pi=\sum_{i} D_{i} \times D_{i}^{\prime}+\gamma \times S
$$

for some divisors $D_{i}, D_{i}^{\prime}$ in $S$ and some 0 -cycle $\gamma$ on $S$. Using the fact that $\pi$ is a projector, it is of the form

$$
\pi=\sum_{i} D_{i} \times D_{i}^{\prime}+\operatorname{deg}(\gamma) \gamma \times S
$$

Now the hypothesis that $\mathrm{CH}^{0}(M)=0$ implies that $\operatorname{deg}(\gamma)=0$; hence, $\pi=\sum_{i} D_{i} \times D_{i}^{\prime}$; that is, $M$ is a direct sum of copies of $\mathbb{1}(-1)$.

\subsection{Todorov surfaces}

Definition 7.3 [46, 66] A Todorov surface is a canonical surface $S$ (i.e., a projective surface with only rational double points as singularities and with $K_{S}$ ample) with $p_{g}(S)=1$, $q=0$, and such that the bicanonical map $\phi_{2 K_{S}}: S \rightarrow \mathbb{P}^{r}$ fits into a commutative diagram 


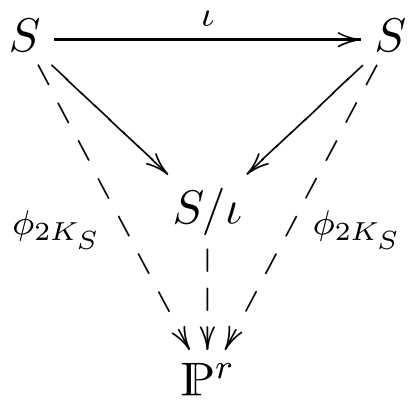

where $\imath: S \rightarrow S$ is an involution for which $S / \imath$ is birational to a K3 surface. The K3 surface obtained by resolving the singularities of $S / \imath$ will be called the K3 surface associated with $S$.

Definition 7.4 [46] The fundamental invariants of a Todorov surface $S$ are $(\alpha, k)$, where $\alpha$ is such that the 2-torsion subgroup of $\operatorname{Pic}(S)$ has order $2^{\alpha}$, and $k=K_{S}^{2}+8$. (The definition of $k$ is explained as the number of rational double points on a so-called distinguished partial desingularization of $S / l$; see [46, Theorem 5.2(ii)].)

Remark 7.5 By Morrison [46, p. 335], there are exactly 11 irreducible families of Todorov surfaces, corresponding to the 11 possible values of the fundamental invariants:

$$
\begin{aligned}
(\alpha, k) \in\{(0,9),(0,10),(0,11),(1,10),(1,11), & \\
& (1,12),(2,12),(2,13),(3,14),(4,15),(5,16)\} .
\end{aligned}
$$

For most of these families (in particular for types $(0,9)$ and $(1,10)$ which are of interest to us), examples of smooth members are given in [66].

\subsection{The Franchetta property for Todorov surfaces of type $(0,9)$ and $(1,10)$}

\subsubsection{Explicit descriptions}

We now restrict attention to Todorov surfaces $S$ with fundamental invariants $(0,9)$ and $(1,10)$. This means that either $K_{S}^{2}=1$ or $K_{S}^{2}=2$ and the fundamental group of $S$ is $\mathbb{Z} / 2 \mathbb{Z}$ [9, Theorem 2.11]. In these two cases, there happens to be a nice explicit description of $S$ in terms of complete intersections in weighted projective spaces.

Theorem 7.6 [8, Theorem 3; 65, Theorem 6] Let $S$ be a Todorov surface with fundamental invariants $(0,9)$. Then $S$ is isomorphic to a complete intersection of two degree 6 hypersurfaces in the weighted projective space $\mathbb{P}:=\mathbb{P}(1,2,2,3,3)$, invariant under the involution

$$
\begin{aligned}
\iota: \mathbb{P} & \rightarrow \mathbb{P} \\
{\left[x_{0}, x_{1}, \ldots, x_{4}\right] } & \mapsto\left[-x_{0}, x_{1}, \ldots, x_{4}\right] .
\end{aligned}
$$


Moreover, the involution a of $\mathbb{P}$ restricts to the involution of Definition 7.3 on $S$.

Conversely, a weighted complete intersection as above with only rational double points as singularities is isomorphic to a Todorov surface with fundamental invariants $(0,9)$.

Theorem 7.7 [9, Theorems 2.8, 2.9] Let $S$ be a Todorov surface with fundamental invariants $(1,10)$. Then $S$ is isomorphic to the quotient $V / \tau$, where $V$ is a complete intersection in the weighted projective space $\mathbb{P}:=\mathbb{P}(1,1,1,2,2)$ having only rational double points as singularities, given by the system of degree 4 equations

$$
\left\{\begin{array}{l}
F=z_{3}^{2}+c w^{4}+w^{2} q\left(x_{1}, x_{2}\right)+Q\left(x_{1}, x_{2}\right)=0 \\
G=z_{4}^{2}+c^{\prime} w^{4}+w^{2} q^{\prime}\left(x_{1}, x_{2}\right)+Q^{\prime}\left(x_{1}, x_{2}\right)=0 .
\end{array}\right.
$$

Here, $\left\lfloor w: x_{1}: x_{2}: z_{3}: z_{4}\right\rfloor$ are coordinates for $\mathbb{P}$, and $q, q^{\prime}$ are quadratic forms, $Q, Q^{\prime}$ are quartic forms without common factor, and $c, c^{\prime}$ are constants not both 0 . The involution $\tau: \mathbb{P} \rightarrow \mathbb{P}$ is defined as

$$
\left[w: x_{1}: x_{2}: z_{3}: z_{4}\right] \mapsto\left[-w: x_{1}: x_{2}: z_{3}: z_{4}\right] .
$$

Moreover, the involution 1 of $\mathbb{P}$ defined as

$$
\left[w: x_{1}: x_{2}: z_{3}: z_{4}\right] \mapsto\left[w: x_{1}: x_{2}:-z_{3}: z_{4}\right]
$$

induces the involution of Definition 7.3 on $S$.

Conversely, given a weighted complete intersection $V \subset \mathbb{P}$ as above, the quotient $S:=V / \tau$ is isomorphic to a Todorov surface with fundamental invariants $(1,10)$.

Remark 7.8 As is shown in [8] (resp. [9]), the Todorov surfaces with fundamental invariants $(0,9)$ (resp. $(1,10))$ form a 12-dimensional subfamily of a larger 18-dimensional (resp. 16-dimensional) family of surfaces of general type with $p_{g}=1, q=0$ and $K^{2}=1$ (resp. $K^{2}=2$ and $\pi_{1}=\mathbb{Z} / 2 \mathbb{Z}$ ), where the above explicit description using complete intersections in the weighted projective space is true. We do not know how to establish an MCK decomposition for these larger families of regular surfaces with $p_{g}=1$. Note that for any

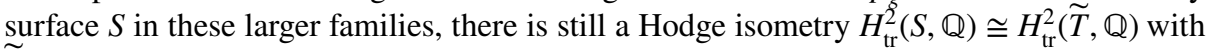
$\widetilde{T}$ a K3 surface [47], but it is not clear whether this isometry is induced by an algebraic correspondence.

\subsubsection{Universal families}

In view of Theorems 7.6 and 7.7, let us construct universal families, which will play a crucial role in establishing the Franchetta property.

Notation 7.9 (i) (Case (0, 9)): Let $\mathbb{P}$ be the weighted projective space $\mathbb{P}(1,2,2,3,3)$. Let $\bar{B} \subset \mathbb{P}\left(\mathrm{H}^{0}\left(\mathbb{P}, \mathcal{O}_{\mathbb{P}}(6)\right)^{\oplus 2}\right)$ be the linear subspace parameterizing pairs of (weighted) homogeneous polynomials of degree 6

$$
F_{b}\left(x_{0}, \ldots, x_{4}\right), \quad G_{b}\left(x_{0}, \ldots, x_{4}\right),
$$

where $x_{0}$ only occurs in even degree. Let $B$ be the Zariski open subset of $\bar{B}$ consisting of $b \in \bar{B}$ such that 


$$
S_{b}:=\left\{x \in \mathbb{P} \mid F_{b}(x)=G_{b}(x)=0\right\}
$$

is a non-singular surface.

Let $\mathcal{S} \rightarrow B$ denote the total space of the family over $B$ (i.e., by Theorem 7.6, the fiber $S_{b}$ over $b \in B$ is a smooth Todorov surface of type $(0,9)$ ), and let $\overline{\mathcal{S}} \rightarrow \bar{B}$ denote the total space of the family over $\bar{B}$. We have a diagram of families

$$
\begin{array}{lll}
\mathcal{S} & \subset \overline{\mathcal{S}} \\
\downarrow & \downarrow \\
\mathcal{T} & \subset \overline{\mathcal{T}}
\end{array}
$$

where $\overline{\mathcal{T}}$ (resp. $\mathcal{T}$ ) is the quotient of $\overline{\mathcal{S}}$ (resp. $\mathcal{S}$ ) under the involution $l$ induced by $\left[x_{0}, x_{1}, \ldots, x_{4}\right] \mapsto\left[-x_{0}, x_{1}, \ldots, x_{4}\right]$. That is, the fiber $T_{b}$ over $b \in B$ is a "singular K3 surface," and its minimal resolution is the K3 surface associated with $S_{b}$.

(ii) (Case $(1,10)$ ): Let $\mathbb{P}$ be the weighted projective space $\mathbb{P}(1,1,1,2,2)$ with coordinates $\left[w, x_{1}, x_{2}, z_{3}, z_{4}\right]$. Let $\bar{B}$ be the linear subspace of $\mathbb{P}\left(\mathrm{H}^{0}\left(\mathbb{P}, \mathcal{O}_{\mathbb{P}}(4)\right)^{\oplus 2}\right)$ parameterizing pairs of weighted homogeneous equations of the form

$$
\left\{\begin{array}{l}
F_{b}=a z_{3}^{2}+c w^{4}+w^{2} q\left(x_{1}, x_{2}\right)+Q\left(x_{1}, x_{2}\right), \\
G_{b}=a^{\prime} z_{4}^{2}+c^{\prime} w^{4}+w^{2} q^{\prime}\left(x_{1}, x_{2}\right)+Q^{\prime}\left(x_{1}, x_{2}\right) .
\end{array}\right.
$$

Let $B$ be the Zariski open subset of $\bar{B}$ consisting of points $b \in \bar{B}$ such that

$$
V_{b}:=\left\{x \in \mathbb{P} \mid F_{b}(x)=G_{b}(x)=0\right\}
$$

is a smooth surface and $F_{b}, G_{b}$ are as in Theorem 7.7 (in particular $a a^{\prime} \neq 0$ and $c, c^{\prime}$ not both 0 ).

Let $\mathcal{V} \rightarrow B$ denote the total space of the family over $B$, and let $\mathcal{S}:=\mathcal{V} /\langle\tau\rangle \rightarrow B$ denote the family obtained by applying the fixed point-free involution $\tau \times \mathrm{id}_{B}$ to $\mathcal{V} \subset \mathbb{P} \times B$ (i.e., by Theorem 7.7, the fiber $S_{b}$ over $b \in B$ is a smooth Todorov surface of type $(1,10)$ ). Denote similarly $\overline{\mathcal{V}}$ and $\overline{\mathcal{S}}:=\overline{\mathcal{V}} /\langle\tau\rangle$ the total spaces of the corresponding families over $\bar{B}$. We have a diagram of families

$$
\begin{array}{llll}
\mathcal{V} & \subset & \overline{\mathcal{V}} \\
\downarrow & & \downarrow \\
\mathcal{S} & \subset \overline{\mathcal{S}} \\
\downarrow & & \downarrow \\
\mathcal{T} & \subset & \overline{\mathcal{T}}
\end{array}
$$

where $\overline{\mathcal{T}}$ (resp. $\mathcal{T}$ ) is the quotient of $\overline{\mathcal{S}}$ (resp. $\mathcal{S}$ ) under the involution $l$ induced by $\left[w, x_{1}, x_{2}, z_{3}, z_{4}\right] \mapsto\left[w, x_{1}, x_{2},-z_{3}, z_{4}\right]$. That is, the fiber $T_{b}$ over $b \in B$ is a "singular K3 surface," and its minimal resolution is the K3 surface associated with $S_{b}$.

Remark 7.10 In both cases of Notation 7.9, it can be checked (cf. [8, 9]) that the parameter space $B$ is non-empty, i.e., the general Todorov surface of type $(0,9)$ or $(1,10)$ is smooth. See also Remark 7.5. 


\subsubsection{The Franchetta property}

Proposition 7.11 (Franchetta property for $T$ ) Let $\mathcal{T} \rightarrow B$ be the universal family as above. Let $\gamma \in \mathrm{CH}^{2}(\mathcal{T})$ be a cycle that has degree 0 on the general fiber. Then

$$
\left.\gamma\right|_{T_{b}}=0 \text { in } \mathrm{CH}^{2}\left(T_{b}\right) \quad \forall b \in B .
$$

Proof For the case $(0,9)$, the family $\mathcal{T} \rightarrow B$ is constructed as the quotient

$$
\pi: \mathcal{S} \rightarrow \mathcal{S} /\langle\imath\rangle=: \mathcal{T}
$$

where $\mathcal{S} \rightarrow B$ is as in Notation 7.9(i), and $\iota$ is as in Theorem 7.6. The quotient $\mathbb{P} /\langle l\rangle$ can be identified with the weighted projective space $\mathbb{P}^{\prime}:=\mathbb{P}(2,2,2,3,3)$, and quotients of the form $T_{b}=S_{b} /\left\langle l_{b}\right\rangle$ can be identified with weighted complete intersections of degree $(6,6)$ in $\mathbb{P}^{\prime}$. It follows that $\overline{\mathcal{T}} \rightarrow \bar{B}$ is the same as the universal family of weighted complete intersections of degree $(6,6)$ in $\mathbb{P}^{\prime}$. As such, $\overline{\mathcal{T}} \rightarrow \mathbb{P}^{\prime}$ has the structure of a projective bundle.

Once we have a projective bundle structure, the argument proving (25) shows the following equality:

$$
\operatorname{Im}\left(\mathrm{CH}^{*}(\overline{\mathcal{T}}) \rightarrow \mathrm{CH}^{*}\left(T_{b}\right)\right)=\operatorname{Im}\left(\mathrm{CH}^{*}\left(\mathbb{P}^{\prime}\right) \rightarrow \mathrm{CH}^{*}\left(T_{b}\right)\right), \quad \forall b \in B .
$$

But $\mathrm{CH}^{2}\left(\mathbb{P}^{\prime}\right)$ is one-dimensional, generated by the square of a hyperplane $h \subset \mathbb{P}^{\prime}$. It follows that for any $\gamma \in \mathrm{CH}^{2}(\mathcal{T})$,

$$
\left.\gamma\right|_{T_{b}}=\left.m h^{2}\right|_{T_{b}} \quad \text { in } \mathrm{CH}^{2}\left(T_{b}\right)
$$

where $m \in \mathbb{Q}$ and $h_{b}:=\left.h\right|_{T_{b}} \in \mathrm{CH}^{1}\left(T_{b}\right)$ is a hyperplane section. This implies the Franchetta property.

For the case $(1,10)$, the family $\mathcal{T} \rightarrow B$ is constructed as the quotient

$$
\pi: \mathcal{V} \rightarrow \mathcal{V} /\langle\tau, \imath\rangle=: \mathcal{T}
$$

where $\mathcal{V} \rightarrow B$ and $\tau$ and $l$ are as in (ii) of Notation 7.9. We note that for any $b \in B$ the surface $V_{b} \subset \mathbb{P}$ is contained in $\mathbb{P}^{-}:=\mathbb{P} \backslash\{[0,0,0,1,0],[0,0,0,0,1]\}$. This means that $\mathcal{V}$ is a Zariski open subset of $\overline{\mathcal{V}}^{-}$, which is defined by the fiber diagram

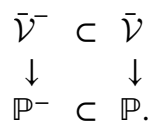

It is proved in [37, Lemma 2.12] that $\overline{\mathcal{V}}^{-} \rightarrow \mathbb{P}^{-}$is a $\mathbb{P}^{r}$-bundle. The argument proving (25) then shows that there is equality

$$
\operatorname{Im}\left(\mathrm{CH}^{*}\left(\overline{\mathcal{V}}^{-}\right) \rightarrow \mathrm{CH}^{*}\left(V_{b}\right)\right)=\operatorname{Im}\left(\mathrm{CH}^{*}\left(\mathbb{P}^{-}\right) \rightarrow \mathrm{CH}^{*}\left(V_{b}\right)\right) \quad \forall b \in B .
$$

But $\mathrm{CH}^{2}\left(\mathbb{P}^{-}\right)$is one-dimensional, generated by the square of a hyperplane $h$. It follows that

$$
\left(\pi_{b}\right)^{*}\left(\left.\gamma\right|_{T_{b}}\right)=\left.\left(\pi^{*} \gamma\right)\right|_{V_{b}}=\left.h^{2}\right|_{V_{b}} \text { in } \mathrm{CH}^{2}\left(V_{b}\right),
$$

and we conclude as before. 
Remark 7.12 The link between Todorov surfaces and K3 surfaces is interesting also from the K3 side. Indeed, Proposition 7.11 says that the Franchetta property holds for the universal K3 surface obtained as a double cover of the projective plane branched along two cubics. (The fact that the quotient $T$ is of this type is proved in [57]. The fact that a general such K3 is quotient of a Todorov surface of type $(0,9)$ is because the period map for Todorov surfaces of type $(0,9)$ is known to have two-dimensional fibers [65].) This corresponds to a ten-dimensional locus inside $\mathcal{F}_{2}$, the moduli stack of K3 surfaces of degree 2 , and it is not a priori clear that the Franchetta property should be true over this locus. This is similar to Remark 6.4 about special cubic fourfolds related to Küchle fourfolds of type $c 7$.

\subsection{Constructing an MCK decomposition}

We prove Theorem 2. First, we recall the following result concerning the Chow group of 0 -cycles of Todorov surfaces of type $(0,9)$ and $(1,10)$. It can be seen as a special case of the Bloch conjecture.

Theorem 7.13 [35, 37] Let $S$ be a smooth Todorov surface with fundamental invariants $(0,9)$ or $(1,10)$. Let $\widetilde{T}$ be its associated $K 3$ surface, and let $\Gamma \in \mathrm{CH}^{2}(S \times \widetilde{T})$ be the correspondence induced by the quotient morphism $S \rightarrow T:=S / l$ and the resolution of singularities $\widetilde{T} \rightarrow T$. Then $\Gamma$ induces an isomorphism

$$
\Gamma_{*}: \mathrm{CH}_{0}(S) \stackrel{\cong}{\longrightarrow} \mathrm{CH}_{0}(\widetilde{T}) .
$$

Proof (Sketch of proof) The $(0,9)$ case is $[35$, Proposition 30], while the $(1,10)$ case is [37, Theorem 5.2]. In both cases, the crux in proving an isomorphism of Chow groups is that there is an explicit description of the surfaces $S$ in terms of (quotients of) complete intersections. This ensures that Voisin's method of "spread" [72] applies. This method exploits the fact that the total space of the universal family of complete intersections of a certain type has a very simple structure. Thanks to this simple structure, one can prove the Franchetta property for self-correspondences of degree zero that exist universally. This applies in particular to (a modification of) the graph of the involution $l$.

Proof (Proof of Theorem 2) This is an application of Proposition 7.1, with $\mathcal{S} \rightarrow B$ the family of smooth Todorov surfaces, and $\mathcal{T} \rightarrow B$ the family of quotients under the involution $t$. Let us ascertain that the hypotheses of Proposition 7.1 are met with. The Franchetta property for $\mathcal{T} \rightarrow B$ is Proposition 7.11. The MCK decomposition for the "singular K3 surface" $T$ follows from the MCK decomposition for its minimal resolution of singularities $\widetilde{T}$ (which is a K3 surface and hence admits an MCK decomposition by Example 4.2) via Remark 4.3 (which still makes sense for surfaces with quotient singularities). Finally, hypothesis (iii) is Theorem 7.13.

Remark 7.14 Theorem 7.13 has recently been proved in [75] for Todorov surfaces with fundamental invariants $(2,12)$. As such, it seems likely that the present approach also works to establish an MCK decomposition for this third family of Todorov surfaces.

Open Access This article is licensed under a Creative Commons Attribution 4.0 International License, which permits use, sharing, adaptation, distribution and reproduction in any medium or format, as long as you give appropriate credit to the original author(s) and the source, provide a link to the Creative Commons 
licence, and indicate if changes were made. The images or other third party material in this article are included in the article's Creative Commons licence, unless indicated otherwise in a credit line to the material. If material is not included in the article's Creative Commons licence and your intended use is not permitted by statutory regulation or exceeds the permitted use, you will need to obtain permission directly from the copyright holder. To view a copy of this licence, visit http://creativecommons.org/licenses/by/4.0/.

\section{References}

1. André, Y.: Une introduction aux motifs (motifs purs, motifs mixtes, périodes), Panoramas et Synthèses, 17. Société Mathématique de France, Paris, 2004. xii+261 pp

2. Beauville, A., Donagi, R.: La variété des droites d'une hypersurface cubique de dimension 4. C. R. Acad. Sci. Paris Sér. I Math. 301(14), 703-706 (1985)

3. Beauville, A.: On the splitting of the Bloch-Beilinson filtration. In: Nagel, J. et al. (eds.) Algebraic Cycles and Motives. London Math. Soc. Lecture Notes, vol. 344. Cambridge University Press, Cambridge (2007)

4. Beauville, A.: Sur l'anneau de Chow d'une variété abélienne. Math. Ann. 273, 647-651 (1986)

5. Beauville, A., Voisin, C.: On the Chow ring of a K3 surface. J. Alg. Geom. 13, 417-426 (2004)

6. Bergeron, N., Li, Z.: Tautological classes on moduli spaces of hyper-Kähler manifolds. Duke Math. J. 168(7), 1179-1230 (2019)

7. Bloch, S., Srinivas, V.: Remarks on correspondences and algebraic cycles. Am. J. Math. 105(5), 12351253 (1983)

8. Catanese, F.: Surfaces with $K^{2}=p_{g}=1$ and their period mapping. In: Algebraic Geometry (Copenhagen, 1978). Springer Lecture Notes in Mathematics. Springer, Berlin (1979)

9. Catanese, F., Debarre, O.: Surfaces with $K^{2}=2, p_{g}=1, q=0$. J. Reine U. Angew. Math. 395, 1-55 (1989)

10. Ceresa, G.: $C$ is not algebraically equivalent to $C^{-}$in its Jacobian. Ann. Math. (2) 117(2), 285-291 (1983)

11. Debarre, O., Voisin, C.: Hyper-Kähler fourfolds and Grassmann geometry. J. Reine Angew. Math. 649, 63-87 (2010)

12. Deninger, C., Murre, J.: Motivic decomposition of abelian schemes and the Fourier transform. J. Reine Angew. Math. 422, 201-219 (1991)

13. Diaz, H.A.: The Chow ring of a cubic hypersurface, I.M.R.N. (2019)

14. Fatighenti, E., Mongardi, G.: Fano varieties of K3 type and IHS manifolds. arXiv:1904.05679

15. Fu, L.: Decomposition of small diagonals and Chow rings of hypersurfaces and Calabi-Yau complete intersections. Adv. Math. 244, 894-924 (2013)

16. Fu, L., Laterveer, R., Vial, Ch.: The generalized Franchetta conjecture for some hyper-Kähler varieties, with a joint appendix with M. Shen. J. Math. Pures Appl. 130, 1-35 (2019)

17. Fu, L., Laterveer, R., Vial, Ch.: The generalized Franchetta conjecture for some hyper-Kähler varieties, II. J. de l'École polytechnique (2021, to appear). arXiv:2002.05490

18. Fu, L., Vial, Ch.: Distinguished cycles on varieties with motive of abelian type and the section property. J. Algebr. Geom. 29, 53-107 (2020)

19. Fu, L., Vial, Ch.: Cubic fourfolds, Kuznetsov components and Chow motives. arXiv:2009.13173

20. Fu, L., Tian, Z., Vial, Ch.: Motivic hyper-Kähler resolution conjecture, I: generalized Kummer varieties. Geom. Topol. 23(1), 427-492 (2019)

21. Fulton, W.: Intersection theory. Springer-Verlag Ergebnisse der Mathematik, Berlin, Heidelberg (1984)

22. Galkin, S., Shinder, E.: The Fano variety of lines and rationality problem for a cubic hypersurface. arXiv: 1405.5154

23. Gross, B., Schoen, C.: The modified diagonal cycle on the triple product of a pointed curve. Ann. Inst. Fourier (Grenoble) 45(3), 649-679 (1995)

24. Iliev, A., Manivel, L.: Fano manifolds of degree 10 and EPW sextics. Ann. Sci. Ecole Norm. Sup. 44, 393-426 (2011)

25. Iliev, A., Manivel, L.: Hyperkähler manifolds from the Tits-Freudenthal square. Eur. J. Math. 5, 11391155 (2019)

26. Iliev, A., Kapustka, G., Kapustka, M., Ranestad, K.: EPW cubes. J. Reine Angew. Math. 748, 241-268 (2019)

27. Izadi, E.: A Prym construction for the cohomology of a cubic hypersurface. Proc. Lond. Math. Soc. 79(3), 535-568 (1999) 
28. Jannsen, U.: Motivic sheaves and filtrations on Chow groups. In: Motives (Jannsen, U. et al. (eds.) Proceedings of Symposia in Pure Mathematics, Part 1, vol. 55 (1994)

29. Kimura, S.-I.: Chow groups are finite dimensional, in some sense. Math. Ann. 331(1), 173-201 (2005)

30. Küchle, O.: On Fano 4-folds of index 1 and homogeneous vector bundles over Grassmannians. Math. Z. 218, 563-575 (1995)

31. Künnemann, K.: On the Chow motive of an Abelian scheme. In: Motives (Seattle, WA, 1991), volume 55 of Proceedings Symposium Pure Mathematics, pp. 189-205. American Mathematical Society, Providence, RI (1994)

32. Kuznetsov, A.: On Küchle varieties with Picard number greater than 1. Izvestiya RAN: Ser. Mat. 79:4 (2015), 57-70 (in Russian); translation in Izvestiya: Mathematics 79:4 (2015), 698-709

33. Kuznetsov, A.: Küchle fivefolds of type $c 5$. Math. Z. 284, 1245-1278 (2016)

34. Kuznetsov, A., Markushevich, D.: Symplectic structures on moduli spaces of sheaves via the Atiyah class. J. Geom. Phys. 59, 843-860 (2009)

35. Laterveer, R.: Some results on a conjecture of Voisin for surfaces of geometric genus one. Boll. Unione Mat. Italiana 9(4), 435-452 (2016)

36. Laterveer, R.: A remark on the motive of the Fano variety of lines of a cubic. Ann. Math. Qué. 41, 141-154 (2017)

37. Laterveer, R.: Algebraic cycles and Todorov surfaces. Kyoto J. Math. 58(3), 493-527 (2018)

38. Laterveer, R.: A remark on the Chow ring of Küchle fourfolds of type d3. Bull. Austral. Math. Soc. 100(3), 410-418 (2019)

39. Laterveer, R.: Algebraic cycles and Verra fourfolds. Tohoku Math. J. 72(3), 451-485 (2020)

40. Laterveer, R.: On the Chow ring of Fano varieties of type S2. Abh. Math. Semin. Univ. Hambg. 90, 17-28 (2020)

41. Laterveer, R.: On the Chow ring of certain Fano fourfolds. Ann. Univ. Paed. Cracoviensis Stud. Math. 19, 39-52 (2020)

42. Laterveer, R., Vial, Ch.: On the Chow ring of Cynk-Hulek Calabi-Yau varieties and Schreieder varieties. Can. J. Math. 72(2), 505-536 (2020)

43. Laza, R., Saccà, G., Voisin, C.: A hyper-Kähler compactification of the intermediate Jacobian fibration associated with a cubic 4-fold. Acta Math. 218, 55-135 (2017)

44. Lehn, Ch., Lehn, M., Sorger, Ch., van Straten, D.: Twisted cubics on cubic fourfolds. J. Reine Angew. Math. 731, 87-128 (2017)

45. Marini, G.: Tautological cycles on Jacobian varieties. Collect. Math. 59(2), 167-190 (2008)

46. Morrison, D.: On the moduli of Todorov surfaces. In: Hijikata, H. et al. (eds.) Algebraic Geometry and Commutative Algebra in Honor of Masayoshi Nagata, vol. 1. Kinokuniya, Tokyo (1988)

47. Morrison, D.: Isogenies between algebraic surfaces with geometric genus one. Tokyo J. Math. 10(1), 179-187 (1987)

48. Murre, J.: On a conjectural filtration on the Chow groups of an algebraic variety, parts I and II. Indag. Math. 4, 177-201 (1993)

49. Neguţ, A., Oberdieck, G., Yin, Q.: Motivic decompositions for the Hilbert scheme of points of a K3 surface. Preprint arXiv:1912.09320

50. O’Grady, K.: Irreducible symplectic 4-folds and Eisenbud-Popescu-Walter sextics. Duke Math. J. 134(1), 99-137 (2006)

51. O'Grady, K.: Moduli of sheaves and the Chow group of K3 surfaces. J. Math. Pures Appl. 100(5), 701-718 (2013)

52. O’Grady, K.: Decomposable cycles and Noether-Lefschetz loci. Doc. Math. 21, 661-687 (2016)

53. Otsubo, N.: On the Abel-Jacobi maps of Fermat Jacobians. Math. Z. 270(1-2), $423-444$ (2012)

54. Otwinowska, A.: Remarques sur les groupes de Chow des hypersurfaces de petit degré. C. R. Acad. Sci. Paris Sér. I Math. 329(1), 51-56 (1999)

55. Pavic, N., Shen, J., Yin, Q.: On O'Grady's generalized Franchetta conjecture. Int. Math. Res. Notices 1-13 (2016)

56. Rieß, U.: On the Chow ring of birational irreducible symplectic varieties. Manuscr. Math. 145(3-4), 473-501 (2014)

57. Rito, C.: A note on Todorov surfaces. Osaka J. Math. 46(3), 685-693 (2009)

58. Schnell, Ch.: Two lectures about Mumford-Tate groups. Rend. Semin. Mat. Univ. Politec. Torino 69(2), 199-216 (2011)

59. Schreieder, S.: On the construction problem for Hodge numbers. Geom. Topol. 19(1), 295-342 (2015)

60. Shen, M., Vial, Ch.: On the motive of the Hilbert cube $X^{[3]}$. Forum Math. Sigma 4 (2016)

61. Shen, M., Vial, Ch.: The Fourier transform for certain hyperKähler fourfolds. Mem. AMS 240(1139) (2016) 
62. Shimada, I.: On the cylinder isomorphism associated to the family of lines on a hypersurface. J. Fac. Sci. Univ. Tokyo Sect. IA Math. 37, 703-719 (1990)

63. Shimada, I.: On the cylinder homomorphisms of Fano complete intersections. J. Math. Soc. Jpn. 42(4), 619-638 (1990)

64. Tavakol, M.: Tautological classes on the moduli space of hyperelliptic curves with rational tails. J. Pure Appl. Algebra 222(8), 2040-2062 (2018)

65. Todorov, A.: Surfaces of general type with $p_{g}=1$ and $(K, K)=1$. Ann. Sci. de l'Ecole Normale Sup. 13, 1-21 (1980)

66. Todorov, A.: A construction of surfaces with $p_{g}=1, q=0$ and $2 \leq\left(K^{2}\right) \leq 8$ : counterexamples of the global Torelli theorem. Invent. Math. 63, 287-304 (1981)

67. Vial, Ch.: Projectors on the intermediate algebraic Jacobians. N. Y. J. Math. 19, 793-822 (2013)

68. Vial, Ch.: Niveau and coniveau filtrations on cohomology groups and Chow groups. Proc. LMS 106(2), 410-444 (2013)

69. Vial, Ch.: Chow-Künneth decomposition for 3- and 4-folds fibred by varieties with trivial Chow group of zero-cycles. J. Algebr. Geom. 24, 51-80 (2015)

70. Vial, Ch.: On the motive of some hyperkähler varieties. J. Reine U. Angew. Math. 725, 235-247 (2017)

71. Voisin, C.: On the Chow ring of certain algebraic hyperkähler manifolds, part 2. Pure Appl. Math. Q. 4(3), 613-649 (2008)

72. Voisin, C.: Chow Rings, Decomposition of the Diagonal, and the Topology of Families. Princeton University Press, Princeton and Oxford (2014)

73. Voisin, C.: On the universal $\mathrm{CH}_{0}$ group of cubic hypersurfaces. J. Eur. Math. Soc. 19(6), 1619-1653 (2017)

74. Yin, Q.: Finite-dimensionality and cycles on powers of K3 surfaces. Comment. Math. Helv. 90, 503$511(2015)$

75. Zangani, N.: Algebraic cycles on Todorov surfaces of type (2,12). arXiv:1905.10123

Publisher's Note Springer Nature remains neutral with regard to jurisdictional claims in published maps and institutional affiliations. 